\title{
Single-cell RNA-Seq Reveals Transcriptional Landscape and Intra-tumor Heterogenicity in Gallbladder Cancer Liver Metastasis Microenvironment
}

\author{
Wenhua You \\ Nanjing Medical University \\ Xiangyu Li \\ Nanjing Medical University \\ Peng Wang \\ Nanjing Medical University \\ Bowen Sha \\ Nanjing Medical University \\ Yuan Liang \\ Nanjing Medical University \\ Jiannan Qiu \\ Nanjing Medical University \\ Jinren Zhou \\ Nanjing Medical University \\ Haoran Hu
}

Nanjing Medical University

\section{Feng Cheng}

Nanjing Medical University

Ling Lu ( $\boldsymbol{\nabla}$ Ivling@njmu.edu.cn )

Jiangsu Key Lab of Collaborative Innovation Center for Personalized Cancer Medicine, Nanjing Medical University, 210046, China.

\section{Research}

Keywords: gallbladder cancer, liver metastasis, single-cell transcriptome, tumor microenvironment

Posted Date: December 7th, 2020

DOI: https://doi.org/10.21203/rs.3.rs-117830/v1 
License: (c) (i) This work is licensed under a Creative Commons Attribution 4.0 International License. Read Full License 


\section{Abstract}

Background: Gallbladder cancer (GBC) is a highly aggressive biliary epithelial malignancy. Tumor invasion and metastasis contributed to the high mortality of GBC patients. However, molecular mechanisms involved in GBC metastases are still little known.

Methods: We performed single-cell RNA sequencing on GBC liver metastasis tissue and analyzed the data based on different cell types.

Results: In this study, 8 cell types, including T cells, B cells, malignant cells, fibroblasts, endothelial cells, macrophages, dendritic cells, and mast cells were identified. Malignant cells displayed a high degree of intra-tumor heterogenicity and neutrophils could promote GBC progression in vitro. Besides, cytotoxic $\mathrm{CD}^{+} \mathrm{T}$ cells became exhausted and $\mathrm{CD} 4^{+}$Tregs presented immunosuppressive characteristics. Macrophages played an important role in the tumor microenvironment. We identified three distinct macrophage subsets and emerged M2 polarization. We also found that cancer-associated fibroblasts exhibited heterogeneity and promoted GBC metastasis.

Conclusions: In conclusion, our work provided a landscape view at the single-cell level and may clear the way for the therapy of GBC metastases.

\section{Background}

Gallbladder cancer (GBC) is a common biliary tract tumor with extremely poor prognosis and high aggressiveness $[1,2]$. The majority of GBC patients have already reached an advanced stage at the time of diagnosis and only $10 \%$ of patients could get access to curative surgery[3]. Among those who underwent surgical resection, recurrence, and metastasis rates remain high. Hepatic invasion and metastatic progression are common and represent a major cause of mortality in GBC patients $[4,5]$. However, the mechanisms of GBC cell metastasis have not yet been elucidated.

The tumor microenvironment (TME) plays an important role in tumor metastasis[6]. The GBC microenvironment consists of malignant cells, stromal cells, and extracellular components. Some factors such as TGF $\beta$, IL-10, and VEGF secreted by cellular elements in the TME contribute to desmoplasia, immunosuppression, and metastasis[7]. The TME has become an attractive target for drug therapy strategies[8].

Single-cell RNA sequencing (scRNA-seq) provided a powerful tool to reveal cellular diversity and disclose microenvironment heterogeneity at single-cell resolution $[9,10]$. In recent years, scRNA-seq has been used to dissect the complicated TME of several cancers such as hepatocellular carcinoma[11, 12], intrahepatic cholangiocarcinoma[13], colorectal cancer[14, 15], glioblastoma[16], and pancreatic ductal adenocarcinoma[17]. However, microenvironment heterogeneity at the single-cell level in human GBC liver metastases is still poorly understood. 
In this study, we utilized scRNA-seq to examine single cells from human GBC liver metastasis tissue and identified 8 cell types corresponding to 7788 cells. Malignant cells presented intra-tumor heterogenicity and neutrophils may play an important role in GBC liver metastases. We proved that neutrophils facilitate GBC cell proliferation, migration, and invasion in vitro. Besides, tumor-infiltrating Tregs exhibited immunosuppressive characteristics and $\mathrm{CD} 8^{+}$cytotoxic $\mathrm{T}$ cells turned to be exhausted. We also identified 3 macrophage subtypes. M2 polarization was common in the TME. Furthermore, RGS5 ${ }^{+}$cancerassociated fibroblasts

Together, we hope this work could help understand tumor microenvironment composition and promote GBC metastasis therapy.

\section{Methods}

\section{Tumor Sample Collection}

Human cancer tissue was obtained from a female patient who was diagnosed as squamous cell carcinoma of gallbladder with liver metastasis. The tissue sample was collected with the approval by the institutional research ethics committee of the First Affiliated Hospital of Nanjing Medical University and informed consent was also signed for the enrolled patient.

\section{Fresh Tissue Preparation and Dissociation into Single Cells}

The fresh tumor tissue was immediately washed with phosphate-buffered saline (PBS) and then conserved in MACS Tissue Storage Solution (\#130-100-008) at $4{ }^{\circ} \mathrm{C}$. Tissue digestion was performed according to the Tumor dissociation Kit user guide (Miltenyi \#130-093-237). Cell suspensions were filtered using a $70 \mu \mathrm{m}$ nylon mesh.

$10 \mu \mathrm{l}$ of cell suspensions were counted by Trypan Blue to determine the concentration of live cells. Then the suspensions were centrifuged at $300 \times \mathrm{g}$ and $4^{\circ} \mathrm{C}$ for $5 \mathrm{~min}$, and the supernatant was discarded.

\section{Library Preparation and Sequencing}

We loaded viable cells that had undergone fluorescence-activated cell sorting (FACS) into a well of a microfluidic chip to generate a complementary deoxyribonucleic acid (cDNA) library using a Chromium Single-Cell Instrument (10X Genomics). Single-cell transcriptomic amplification and library preparation were performed by single-cell 3' Reagent Kit v3 (10X Genomics) according to the manufacturer's protocol. The library was loaded on an Illumina XTEN system.

\section{Single Cell Sequencing Data Preprocessing, Quality Control and Subcluster Detection}

The CellRanger software (v3.1.0) was used to process raw FASTQ files, align the sequencing reads to the GRCh38 reference, and generate a filtered feature-barcode matrix. The Seurat R package (v3.1.5)[18] was used to analyse our data. Cells with over 10000 genes and more than $25 \%$ of mitochondrial UMI counts 
were removed. The remaining high-quality cells were normalized and scaled. High variable genes were calculated using FindVariableFeatures method with default parameters. The selected features were used for principal components analysis (PCA) analysis. Clusters were found using FindClusters

function(resolution $=0.8$ ). The t-Distributed Stochastic Neighbor Embedding (tSNE) analysis was used for dimension reduction and visualization of gene expression.

\section{Cell Type Classification}

We conducted tSNE dimensionality reduction with the first 50 principal components.

Cell clusters were annotated to known biological cell types using canonical marker genes and the putative CNV score.

\section{Copy Number Variation (CNV) Inferring}

We used the inferCNV (v1.2.1) R package to estimate the CNV score for each single cell. All T cells were served as the reference for inferCNV. The CNV score of each cell was calculated as quadratic sum of CNV region.

\section{Estimate the Transcriptional Diversity of Each Cell}

CytoTRACE (Cellular (Cyto) Trajectory Reconstruction Analysis using gene Counts and Expression) is a computational framework to predict the relative differentiation state of single cell[19]. We used the $R$ software (v0.1.0) to predict the transcriptional diversity.

\section{Pseudotime Trajectory Analysis}

Trajectory analysis was performed using R package Monocle 2 (v2.14.0)[20]. The differentialGeneTest function was used to identify significant genes (qval $<0.01$, num_cells_expressed $\geq 10$ ). Then cell ordering was conducted on these genes. The trajectories were visualized using DDRTree method.

\section{Cell-Cell Communication Analysis}

CellPhoneDB is a python-based tool to analysis cell-cell communication at the molecular level[21]. We used CellPhoneDB python package (v2.1.1) to analyze the potential interaction networks of the 8 major cell types.

\section{Gene Regulatory Network Analysis}

As described previously, we utilized SCENIC R package (v1.1.2-2) to infer gene regulatory networks and cell types from single-cell RNA-seq data[22]. Two gene-motif rankings including $10 \mathrm{~kb}$ around the transcription start site (TSS) and 500 bp upstream of the TSS were used to determine the search space around the TSS. 20-thousand motif database was downloaded for RcisTarget and GENIE3.

\section{Functional Enrichment Analysis}

DEGs were identified using FindAllMarkers function of Seurat. The cutoff threshold values were used: adj.p.val $<0.05$, min.pct $>0.25$ and log fold change $>0.25$. 
R package clusterProfiler[23] was used to perform the Gene Ontology (GO) enrichment analysis. Pathways which the adjusted $p$ value $<0.05$ were considered significantly enriched. Gene set variation analysis (GSVA) was performed in the GSVA R package (v1.34.0)[24] to evaluate pathway activity of 50 hallmark gene sets.

\section{Cell Culture}

The GBC cell line GBC-SD (RRID: CVCL_6903) was purchased from the Procell Life Science \& Technology (Wuhan, China). The cell line has been authenticated using STR (or SNP) profiling within the last three years. The cell line was cultured at RPMI 1640 containing $2 \%$ fetal bovine serum (FBS). All experiments were performed with mycoplasma-free cells.

\section{Isolation and Preparation of Human Neutrophils}

Human neutrophils were isolated from the peripheral blood of healthy donors collected at the First Affiliated Hospital of Nanjing Medical University using Human neutrophils separation kit (Solarbio, Beijing, China). All donors signed the informed consents. Neutrophils were resuspended in RPMI 1640 containing $2 \%$ fetal bovine serum (FBS). Neutrophil-conditioned medium (NCM) was obtained as previously described[25].

\section{Cell Proliferation Assay, Cell Migration and Invasion Assays}

The CCK-8 Cell Counting Kit (Vazyme Biotech Co., Ltd) was utilized to assess the abilities of cell proliferation according to the manufacturer's protocol. In brief, we planted GBC-SD cell line with or without NCM into $96-$ well plates ( 2000 cells/well) with addition of CCK-8 solution ( $10 \mu \mathrm{l} /$ well). After incubation for 2 hours in the incubator described as above, the absorbance at $450 \mathrm{~nm}$ was measured to determine the number of variable cells in each well.

Cell migration was assessed through the scratch wound assay. We made a uniform linear scratch using a 10- $\mu$ l pipet tip on the GBC-SD cell lines which have been cultured with or without NCM for 2 days. The distance between the wound edges was taken as a baseline. Then we acquired the image of the same location after $48 \mathrm{~h}$. The change of the distance was measured to assess the cellular ability of migration.

The 24-well transwell chambers (Millipore, USA) were used to evaluate the invasive capabilities of the cells. Totally, $1 \times 10^{5}$ cells were seeded in the upper chamber. The lower chamber was filled with $100 \mu \mathrm{L}$ RPMI 1640 with $2 \%$ FBS or NCM. After incubation for 24 hours, the cells in the upper chamber were removed using cotton swabs. Cells on the lower surface of membrane were fixed and stained with crystal violet. The GBC cells were visualized under a inverted light microscope (Olympus, Japan), photographed, and counted in five microscopic fields.

All experiments were performed three times.

\section{Statistical Analysis}


All statistical analysis was performed using R platform (v3.6.0). $P<0.05$ was considered statistically significant. Statistical differences between multiple groups were compared using Student's t-test or analysis of variance (ANOVA).

\section{Results}

\section{Single Cell Transcriptomic Analysis Reveals the Cellular Diversity in Gallbladder Cancer (GBC) Liver Metastasis}

To explore the potential mechanisms and microenvironment of GBC liver metastasis, we generated single-cell transcriptomic profile from a GBC liver metastasis tissue using 10X Genomics (Fig. 1A). A total of 7788 cells passed quality control. 8 distinct cell types were identified (Fig. 1B and Supplemental Fig. 1) using the following conventional markers: malignant cells (925 cells, marked with EPCAM and KRT19); T cells (4258 cells, marked with $C D 2, C D 3 D, C D 3 E$, and $C D 3 G$ ); B cells (907 cells, marked with $C D 79 A$ and MS4A1); Macrophages (1214 cells; marked with CD68, CD163, and CD14); Fibroblasts(264 cells, marked with ACTA2 and COL 1A2); Dendritic cells(DC) (79 cells, marked with IL3RA and LILRA4); Endothelial cells(64 cells, marked with VWF, ENG, and CD34) and Mast cells(77 cells, marked with KIT and TPSAB1). The dot plots of marker genes were shown in Fig. 1C. Further copy number variation (CNV) analysis was conducted to distinguish malignant and non-malignant cells (Fig. 1D and Supplemental Fig. 2). The differentially expressed genes (DEGs) in 8 cell types, as shown in Fig. 1F, confirmed the accuracy of cell recognition.

\section{Transcriptomic Intra-tumor Heterogeneity of Tumor Cells in the GBC Liver Metastasis}

In this study, we identified 7 subclusters after the reclustering of malignant cells (Fig. 2A). To investigate the heterogeneity of tumor cells, we used CytoTRACE to evaluate the transcriptional diversity of each cell which is a hallmark of developmental potential. As shown in Fig. 2B(left), the CytoTRACE score was distributed diversely indicating that tumor cells were in different differentiation states. Monocle 2 was used to construct a trajectory. Consistent with the CytoTRACE result, we noticed that the trajectory's root was populated by the majority of M0 cells (high CytoTRACE score). M3 and M4 cells (low CytoTRACE score) were present at the end of two differentiation branches (Fig. 2B right and Supplemental Fig. 3A). In addition, pathway analysis by gene set variation analysis (GSVA) revealed that hallmark pathways were partially activated in subpopulations M0, M1, or M2 with higher CytoTRACE scores, respectively. Particularly, the M4 subcluster had the lowest GSVA score (Fig. 2C). We conducted gene function enrichment analysis on DEGs of M0, M3, and M4 subclusters. As shown in Fig. 2D and Supplemental Fig. 3B, G2M/S cell cycle-related genes were enriched in M0. In M3, neutrophils-associated immune response and IFN-gamma signaling were commonly observed. Besides, the M4 cluster was mainly correlated with nucleocytoplasmic transport pathways. Not only in M3 but also in M2 and M5, neutrophils-related gene functions were enriched suggesting that there is a lot of crosstalk between 
neutrophils and cancer cells during the metastasis of gallbladder cancer (Supplemental Fig. 3C-F). Different functional patterns of tumor cells may reflect diverse processes of the metastatic seeds adapted to the soil. In conclusion, all these results showed a high degree of intra-tumor heterogeneity in malignant cells of GBC metastasis.

\section{Neutrophils promote proliferation, migration and invasion of GBC cells in vitro}

Significant neutrophils infiltration has been observed in human cancer metastatic sites. To examine the effect of neutrophils on GBC cells, we incubated the GBC cell line (GBC-SD) with NCM. CCK-8 proliferation analysis showed that NCM promoted GBC cell growth in vitro (Fig. 3A). Wound-healing migration assays and cell invasion assays also demonstrated that NCM significantly enhanced the migration and invasion abilities of GBC cells (Fig. 3B and C).

\section{Immunosuppressive Tumor-infiltrating Tregs and Exhausted $\mathrm{CD}^{+} \mathrm{T}$ cells are Enriched in the Metastatic Tumor}

T cells were found to be the most prevalent cell type in the tumor microenvironment (TME) of liver metastasis tumor. The tumor-infiltrating immune cells were considered to play an important role in response to immunotherapy. As shown in Fig. 4A, T cells were clustered into 9 distinct subgroups. According to the known markers of T cells, 9 subclusters were annotated as T0-CD8TGZMB $\left(C D 8 A^{+} G Z M B^{+}\right.$, cluster 0, 4), T1-CD8T-GZMK(CD8A $G Z M K^{+}$, cluster 1), T2-Treg-FOXP3 $\left(C D 4^{+}\right.$ FOXP3 ${ }^{+}$, cluster 2), T3-CD4T-naïve $\left(C D 4^{+} I L 7 R^{+}\right.$, cluster 3,7$)$, T4-CD8T-proliferation $\left(C D 8 A^{+} M K I 67^{+}\right.$, cluster $5,8)$, T5-CD8T- naïve $\left(C D 8 A^{+} I L 7 R^{+}\right.$,cluster 6;Figure 4B and $\left.4 D\right)$. In this study, we found that $C D 8^{+} T$ cells (cluster $0,1,4,5,6,8)$ expressed different levels of cytotoxic markers like GZMK, IFNG, and PRF1. In proliferating $\mathrm{CD}^{+} \mathrm{T}$ cell populations (cluster 5 and 8), a set of $\mathrm{T}$ cell exhaustion-related immune checkpoints including TIGIT, LAG3, HAVCR2(TIM3), and CTLA4 tend to be upregulated indicating that the $\mathrm{CD} 8^{+} \mathrm{T}$ cells became exhausted. We utilized Monocle 2 to perform pseudotime analysis on $\mathrm{CD} 8^{+} \mathrm{T}$ cells.

The trajectory was visualized as a DDRTree plot (Fig. 4E). We can notice that naïve T cells tend to convert into proliferating $\mathrm{CD} 8^{+} \mathrm{T}$ cell subgroups which have been become exhausted suggesting that cytotoxic $\mathrm{CD}^{+} \mathrm{T}$ cells were turning to be exhausted. In addition, Tregs (cluster 2) expressed a certain number of immunosuppression markers such as TIGIT, CTLA4, and TNFRSF18(GITR). Interestingly, the expression $\angle A G 3$ and HAVCR2 were found to be highly correlated with the activity of $C D 8^{+} T$ cells which was measured by the average expression of granzymes (GZMA, GZMB and GZMH) (Fig. 4F). Taken together, our analysis showed that in the GBC metastatic tumor environment, cytotoxic $T$ cells tend to be exhausted and Tregs present a highly immunosuppressive phenotype. LAG3 and HAVCR2 may be a potential treatment target for patients with GBC liver metastasis. 


\section{Macrophages Have More Crosstalk with Malignant Cells and Tend to generate M2-type polarization}

We used CellPhoneDB to infer intercellular communication in the TME. Macrophages were found to have more interactions with tumor cells, revealing an important role in the microenvironment (Fig. 5A). To investigate the heterogeneity of macrophages, we clustered macrophages into 3 subclusters (Fig. 5B). As shown in Fig. 5C, subcluster 0 cells highly expressed M2-type macrophage markers including CD163, $A P O E$, and MAF. Cells from cluster 1 exhibited the M1-like phenotype. SPP1 also known as secreted phosphoprotein 1 was also expressed in subcluster 1. It has been reported that pro-inflammatory molecules such as interferon- gamma and interleukin-1 $\beta$ could induce SPP1 expression which plays an important role in colon cancer liver metastasis in previous studies[26]. Figure 5D shows the expression of SPP1 in liver hepatocellular carcinoma ( $\mathrm{LIHC})$ and cholangiocarcinoma (CHOL) for the lack of GBC expression data. SPP1 was highly expressed in tumor tissues and correlated with overall survival in LIHC (Supplemental Fig. 4A) and disease-free survival in CHOL (Supplemental Fig. 4B). In addition, MKI67 and STMN1 which correlated with cell cycle were expressed by cells from subcluster 2. GSVA revealed that interferon (IFN) pathways were enriched in subcluster 0, however, the inflammatory response was also activated (Fig. 5F). SCENIC analysis showed that genes regulated by FOS, JUN, STAT1, ERG1, and RUNX1 were upregulated in subcluster 0 (Fig. 5E). Fos/Jun has been reported to be able to enhance inflammatory responses in macrophages. IRF7, which was activated in Macrophage subcluster 1, participates in the regulation of the M1-to-M2 phenotype switch[27]. In conclusion, M2-like macrophages remained some pro-inflammatory functions. Furthermore, trajectory analysis showed that M2-like macrophages were present at the end of the differentiation trajectory (Fig. 5G). All evidence supported the $\mathrm{M} 2$ polarization phenomenon in GBC metastasis.

\section{Fibroblasts Exhibit Heterogeneity and promote GBC progression}

As shown in Fig. 6A and $\mathrm{B}$, cancer-associate fibroblasts (CAFs) from three subgroups were almost positive for a-SMA (ACTA2) which is a classical marker of fibroblasts. These three subgroups expressed different markers (cluster 0: $\mathrm{MAFB}^{+}, \mathrm{GPB}^{+}$; cluster 1: $\mathrm{RGS5}^{+}, \mathrm{SMOC2}^{+}$; cluster 2: $\mathrm{ACTG2} 2^{+}, \mathrm{CCL} 11^{+}$; Fig. 6C). The GO analysis results of DEGs in three distinct subgroups were shown in Supplemental Fig. 5. $R G S 5$, a marker for vascular CAFs (VCAFs), has been reported to be related to invasion and metastasis of cancers such as liver cancer and lung cancer by inducing EMT [28, 29]. The SCENIC analysis revealed that genes regulated by TF NR2F2 and NFIL3 were upregulated in cells from the RGS5 ${ }^{+}$fibroblasts subgroup (Fig. 6D). Previous studies have proved that NR2F2 (COUP-TFII) is a transcription factor closely associated with tumorigenesis, invasion and metastasis in several digestive cancer such as pancreatic[30], colorectal[31], and gastric cancer[32]. NFIL3 also known as E4-binding protein 4 (E4BP4) is a leucine zipper transcription factor in the immune system. Lin et al. found that cellular prion protein transcriptionally regulated by NFIL3 promotes lung cancer invasion and metastasis[33]. In conclusion, all 
these data suggested that RGS5 ${ }^{+}$fibroblasts may contribute to the tumorigenesis, invasion, and metastasis of GBC.

\section{Discussion}

GBC is a highly aggressive and lethal tumor[34]. Currently, the therapy of GBC, especially metastatic GBC, is still a challenge[2, 3,34]. Only a few patients could benefit from curative resection, however, the prognosis is poor. The mechanisms underlying GBC metastasis are little known. Several studies showed that TME participates in tumor progression and metastasis. Disrupting the pro-tumorigenic TME is a promising therapeutic target for cancer patients. In this study, we employed scRNA-seq to delineate the transcriptomic heterogeneity of the TME in GBC liver metastasis at single-cell resolution.

In this work, several observations were found by describing intra-tumoral heterogeneity of the TME in GBC liver metastasis. First, GBC cells exhibit high heterogeneity at the metastatic site. The existence of cell subpopulations in different differentiation states fully reflects the complexity and diversity of GBC in its evolution. In previous studies, neutrophil-to-lymphocyte ratio (NLR) showed prognostic significance in GBC patients[35] and increasing neutrophil levels have a significant influence on the TME by inducing various cytokines and chemokines which could accelerate the proliferation and promote metastasis of tumor cells[36, 37]. Targeting neutrophils might become a new direction for the treatment of GBC metastasis. By pseudotime analysis and GO analysis, neutrophils-associated gene ontology was enriched in the intermediate stage of differentiation trajectory indicating that tumor cells communicate extensively with immune cells at the intermediate stage of metastasis. We also validate the influence of neutrophils on GBC cells in vitro. All evidence proved that targeting tumor-associated neutrophils is a promising method to antitumor metastasis for GBC patients.

Second, the high expression of TIGIT, CTLA4, LAG3, and HAVCR2 in different T cells indicated a tumorsuppressive TME in GBC metastasis. Tregs are immunosuppressive $T$ cells that participate in immune escape and block the antitumor immune response for cancer patients[38]. A set number of Tregs in GBC liver metastasis were observed. The infiltrating level of Tregs is correlated with the disease progression of GBC patients[39, 40]. Besides, LAG3 and HAVCR2, known as exhausted markers, were found to be correlated with $C D 8^{+} T$ cells activity suggesting that $L A G 3$ and HAVCR2 are the most prominent immune checkpoint molecules in GBC metastasis.

Third, three types of macrophages were identified from the TME. M1-like macrophages exhibit $\mathrm{CD} 68^{+} \mathrm{CD} 163^{+} \mathrm{SPP} 1^{+}$phenotype while M2-like macrophages exhibit $\mathrm{CD} 68^{+} \mathrm{IL} 1 \mathrm{~B}^{+} \mathrm{S} 100 \mathrm{~A} 8^{+}$phenotype. M2 polarization was common in the TME.

By GSVA pathway analysis and SCENIC analysis, we revealed some underlying TFs and potential mechanisms involved in the M2 polarization phenomenon. Besides, transcription factor IRF8 was also found activated in the proliferative macrophage population. IRF8 could repress macrophage-derived matricellular protein osteopontin and make it become a novel immunosuppressive checkpoint[41]. 
Osteopontin controls immunosuppression in the tumor microenvironment[42]. Interestingly, M1-like macrophages highly expressed secreted phosphoprotein 1 indicating that SPP $1^{+} \mathrm{M} 1$-like macrophages are correlated with immunosuppression and may be associated with immunotherapy outcomes for patients with GBC metastasis.

Finally, several TFs related to metastasis were found to be activated in RGS5 ${ }^{+}$fibroblasts via SCENIC analysis. A previous study indicated that RGS5 ${ }^{+} \mathrm{CAFs}$ (VCAFs) contribute to tumor progression in intrahepatic cholangiocarcinoma[13]. The data supported that RGS5 ${ }^{+} \mathrm{CAFs}$ is also a key factor in GBC progression.

\section{Conclusions}

Our findings provide a landscape of single-cell expression profiles in GBC liver metastasis and confirmed characteristics of cell subpopulations. We hope this study help address microenvironment-mediated GBC progression and drug resistance while identify novel therapeutic targets for GBC metastasis.

\section{Abbreviations}

GBC

Gallbladder cancer

scRNA-seq

Single-cell RNA sequencing

TME

tumor microenvironment

CNV

copy number variation

TF

transcription factors

DEGs

differentially expressed genes

GSVA

Gene set variation analysis

FBS

fetal bovine serum

PBS

phosphate-buffered saline

NCM

Neutrophil-conditioned medium

LIHC

liver hepatocellular carcinoma 
CHOL

cholangiocarcinoma

CAFs

cancer-associate fibroblasts

NLR

neutrophil-to-lymphocyte ratio

\section{Declarations}

\section{Ethics approval and consent to participate}

The ethic approval has been accepted from the institutional research ethics committee of the First Affiliated Hospital of Nanjing Medical University.

\section{Consent for publication}

Not applicable.

\section{Availability of data and materials}

All data that obtained and analyzed during our study are available from the corresponding author once reasonably requested.

\section{Competing interests}

All authors of this study confirm that they have absolutely no conflicts of interest concerning this manuscript.

\section{Funding}

This work was supported by funds from National Natural Science Foundation of China (81971495, 81571564, 81522020), CAMS Innovation Fund for Medical Sciences (No.2019-I2M-5-035), the National Science Foundation of Jiangsu Province (BRA2017533, BK20131024 and BE2016766), the 863 Young Scientists Special Fund (SS2015AA0209322) and the Foundation of Jiangsu Collaborative Innovation Center of Biomedical Functional Materials.

\section{Authors' Contributions}

WY, XL, PW and BS contributed to study concept and design, perform experiment and data analysis, literature search, make figures and writing of the manuscript. YL, JZ, JQ and HH contributed to data search and analysis. LL and FC contributed to study design, literature search and review of the manuscript. All authors have approved the final version of this paper.

\section{Acknowledgements}




\section{References}

1. Jemal A, Siegel R, Xu J, Ward E: Cancer statistics, 2010.CA Cancer J Clin 2010, 60:277-300.

2. Kanthan R, Senger JL, Ahmed S, Kanthan SC: Gallbladder Cancer in the 21st Century.J Oncol 2015, 2015:967472.

3. Zhu AX, Hong TS, Hezel AF, Kooby DA: Current management of gallbladder carcinoma.Oncologist 2010, 15:168-181.

4. Wullstein C, Woeste G, Barkhausen S, Gross E, Hopt UT: Do complications related to laparoscopic cholecystectomy influence the prognosis of gallbladder cancer? Surg Endosc 2002, 16:828-832.

5. Bartlett DL, Fong Y, Fortner JG, Brennan MF, Blumgart LH: Long-term results after resection for gallbladder cancer. Implications for staging and management.Ann Surg 1996, 224:639-646.

6. Quail DF, Joyce JA: Microenvironmental regulation of tumor progression and metastasis. Nat Med 2013, 19:1423-1437.

7. Ren B, Cui M, Yang G, Wang H, Feng M, You L, Zhao Y: Tumor microenvironment participates in metastasis of pancreatic cancer.Mol Cancer 2018, 17:108.

8. Roma-Rodrigues C, Mendes R, Baptista PV, Fernandes AR: Targeting Tumor Microenvironment for Cancer Therapy.Int J Mol Sci 2019, 20.

9. Levitin HM, Yuan J, Sims PA: Single-Cell Transcriptomic Analysis of Tumor Heterogeneity.Trends Cancer 2018, 4:264-268.

10. Baslan T, Hicks J: Unravelling biology and shifting paradigms in cancer with single-cell sequencing. Nat Rev Cancer 2017, 17:557-569.

11. Ma L, Hernandez MO, Zhao Y, Mehta M, Tran B, Kelly M, Rae Z, Hernandez JM, Davis JL, Martin SP, et al: Tumor Cell Biodiversity Drives Microenvironmental Reprogramming in Liver Cancer.Cancer Cell 2019, 36:418-430 e416.

12. Zhang Q, He Y, Luo N, Patel SJ, Han Y, Gao R, Modak M, Carotta S, Haslinger C, Kind D, et al: Landscape and Dynamics of Single Immune Cells in Hepatocellular Carcinoma.Cel/ 2019, 179:829845 e820.

13. Zhang M, Yang H, Wan L, Wang Z, Wang H, Ge C, Liu Y, Hao Y, Zhang D, Shi G, et al: Single-cell transcriptomic architecture and intercellular crosstalk of human intrahepatic cholangiocarcinoma.J Hepatol 2020.

14. Zhang Y, Song J, Zhao Z, Yang M, Chen M, Liu C, Ji J, Zhu D: Single-cell transcriptome analysis reveals tumor immune microenvironment heterogenicity and granulocytes enrichment in colorectal cancer liver metastases. Cancer Lett 2020, 470:84-94.

15. Li H, Courtois ET, Sengupta D, Tan Y, Chen KH, Goh JJL, Kong SL, Chua C, Hon LK, Tan WS, et al: Reference component analysis of single-cell transcriptomes elucidates cellular heterogeneity in human colorectal tumors. Nat Genet 2017, 49:708-718. 
16. Patel AP, Tirosh I, Trombetta JJ, Shalek AK, Gillespie SM, Wakimoto H, Cahill DP, Nahed BV, Curry WT, Martuza RL, et al: Single-cell RNA-seq highlights intratumoral heterogeneity in primary glioblastoma.Science 2014, 344:1396-1401.

17. Peng J, Sun BF, Chen CY, Zhou JY, Chen YS, Chen H, Liu L, Huang D, Jiang J, Cui GS, et al: Single-cell RNA-seq highlights intra-tumoral heterogeneity and malignant progression in pancreatic ductal adenocarcinoma.Cell Res 2019, 29:725-738.

18. Stuart T, Butler A, Hoffman P, Hafemeister C, Papalexi E, Mauck WM, 3rd, Hao Y, Stoeckius M, Smibert P, Satija R: Comprehensive Integration of Single-Cell Data.Cell 2019, 177:1888-1902 e1821.

19. Gulati GS, Sikandar SS, Wesche DJ, Manjunath A, Bharadwaj A, Berger MJ, llagan F, Kuo AH, Hsieh RW, Cai S, et al: Single-cell transcriptional diversity is a hallmark of developmental potential.Science 2020, 367:405-411.

20. Qiu X, Hill A, Packer J, Lin D, Ma YA, Trapnell C: Single-cell mRNA quantification and differential analysis with Census. Nat Methods 2017, 14:309-315.

21. Efremova M, Vento-Tormo M, Teichmann SA, Vento-Tormo R: CellPhoneDB: inferring cell-cell communication from combined expression of multi-subunit ligand-receptor complexes.Nat Protoc 2020, 15:1484-1506.

22. Aibar S, Gonzalez-Blas CB, Moerman T, Huynh-Thu VA, Imrichova H, Hulselmans G, Rambow F, Marine JC, Geurts P, Aerts J, et al: SCENIC: single-cell regulatory network inference and clustering.Nat Methods 2017, 14:1083-1086.

23. Yu G, Wang LG, Han Y, He QY: clusterProfiler: an R package for comparing biological themes among gene clusters.OMICS 2012, 16:284-287.

24. Hanzelmann S, Castelo R, Guinney J: GSVA: gene set variation analysis for microarray and RNA-seq data.BMC Bioinformatics 2013, 14:7.

25. Zhou SL, Dai Z, Zhou ZJ, Chen Q, Wang Z, Xiao YS, Hu ZQ, Huang XY, Yang GH, Shi YH, et al: CXCL5 contributes to tumor metastasis and recurrence of intrahepatic cholangiocarcinoma by recruiting infiltrative intratumoral neutrophils. Carcinogenesis 2014, 35:597-605.

26. Lee HO, Hong Y, Etlioglu HE, Cho YB, Pomella V, Van den Bosch B, Vanhecke J, Verbandt S, Hong H, Min JW, et al: Lineage-dependent gene expression programs influence the immune landscape of colorectal cancer.Nat Genet 2020, 52:594-603.

27. Cohen M, Matcovitch O, David E, Barnett-Itzhaki Z, Keren-Shaul H, Blecher-Gonen R, Jaitin DA, Sica A, Amit I, Schwartz M: Chronic exposure to TGFbeta1 regulates myeloid cell inflammatory response in an IRF7-dependent manner.EMBO J 2014, 33:2906-2921.

28. Hu M, Chen X, Zhang J, Wang D, Fang X, Wang X, Wang G, Chen G, Jiang X, Xia H, Wang Y: Overexpression of regulator of $\mathrm{G}$ protein signaling 5 promotes tumor metastasis by inducing epithelialmesenchymal transition in hepatocellular carcinoma cells.J Surg Oncol 2013, 108:192-196.

29. Xu Z, Zuo Y, Wang J, Yu Z, Peng F, Chen Y, Dong Y, Hu X, Zhou Q, Ma H, et al: Overexpression of the regulator of G-protein signaling 5 reduces the survival rate and enhances the radiation response of human lung cancer cells.Oncol Rep 2015, 33:2899-2907. 
30. Polvani S, Tarocchi M, Tempesti S, Mello T, Ceni E, Buccoliero F, D'Amico M, Boddi V, Farsi M, Nesi S, et al: COUP-TFIl in pancreatic adenocarcinoma: clinical implication for patient survival and tumor progression.Int J Cancer 2014, 134:1648-1658.

31. Bao Y, Gu D, Feng W, Sun X, Wang X, Zhang X, Shi Q, Cui G, Yu H, Tang C, Deng A: COUP-TFII regulates metastasis of colorectal adenocarcinoma cells by modulating Snail1.Br J Cancer 2014, 111:933-943.

32. Ding W, Zhang Y, Cai H, Liu G, Ye Y, Xu G, Wang H, Xiong D, Zhang C, Huang Z, Luo Q: Overexpression of COUPTFII suppresses proliferation and metastasis of human gastric cancer cells.Mol Med Rep 2018, 17:2393-2401.

33. Lin SC, Lin CH, Shih NC, Liu HL, Wang WC, Lin KY, Liu ZY, Tseng YJ, Chang HK, Lin YC, et al: Cellular prion protein transcriptionally regulated by NFIL3 enhances lung cancer cell lamellipodium formation and migration through JNK signaling. Oncogene 2020, 39:385-398.

34. Misra S, Chaturvedi A, Misra NC, Sharma ID: Carcinoma of the gallbladder.Lancet Oncol 2003, 4:167176.

35. Zhang L, Wang R, Chen W, Xu X, Dong S, Fan H, Liu C: Prognostic significance of neutrophil to lymphocyte ratio in patients with gallbladder carcinoma.HPB (Oxford) 2016, 18:600-607.

36. Gregory AD, Houghton AM: Tumor-associated neutrophils: new targets for cancer therapy.Cancer Res 2011, 71:2411-2416.

37. Liu K, Wang FS, Xu R: Neutrophils in liver diseases: pathogenesis and therapeutic targets.Cell Mol Immunol 2020.

38. Togashi Y, Shitara K, Nishikawa H: Regulatory T cells in cancer immunosuppression - implications for anticancer therapy.Nat Rev Clin Oncol 2019, 16:356-371.

39. Patil RS, Shah SU, Shrikhande SV, Goel M, Dikshit RP, Chiplunkar SV: IL17 producing gammadeltaT cells induce angiogenesis and are associated with poor survival in gallbladder cancer patients.Int $J$ Cancer 2016, 139:869-881.

40. Ozgur HH, Ercetin AP, Eliyatkin N, Seren A, Kupelioglu A, Ortac R, Diniz G, Aktas S: Regulatory T cells and their prognostic value in hepatopancreatobiliary tumours. Hepatogastroenterology 2014, 61:1847-1851.

41. Klement JD, Paschall AV, Redd PS, Ibrahim ML, Lu C, Yang D, Celis E, Abrams SI, Ozato K, Liu K: Repression of osteopontin by IRF8 regulates a novel immunosuppressive checkpoint. The Journal of Immunology 2019, 202:195.126-195.126.

42. Shurin MR: Osteopontin controls immunosuppression in the tumor microenvironment.J Clin Invest 2018, 128:5209-5212.

\section{Figures}




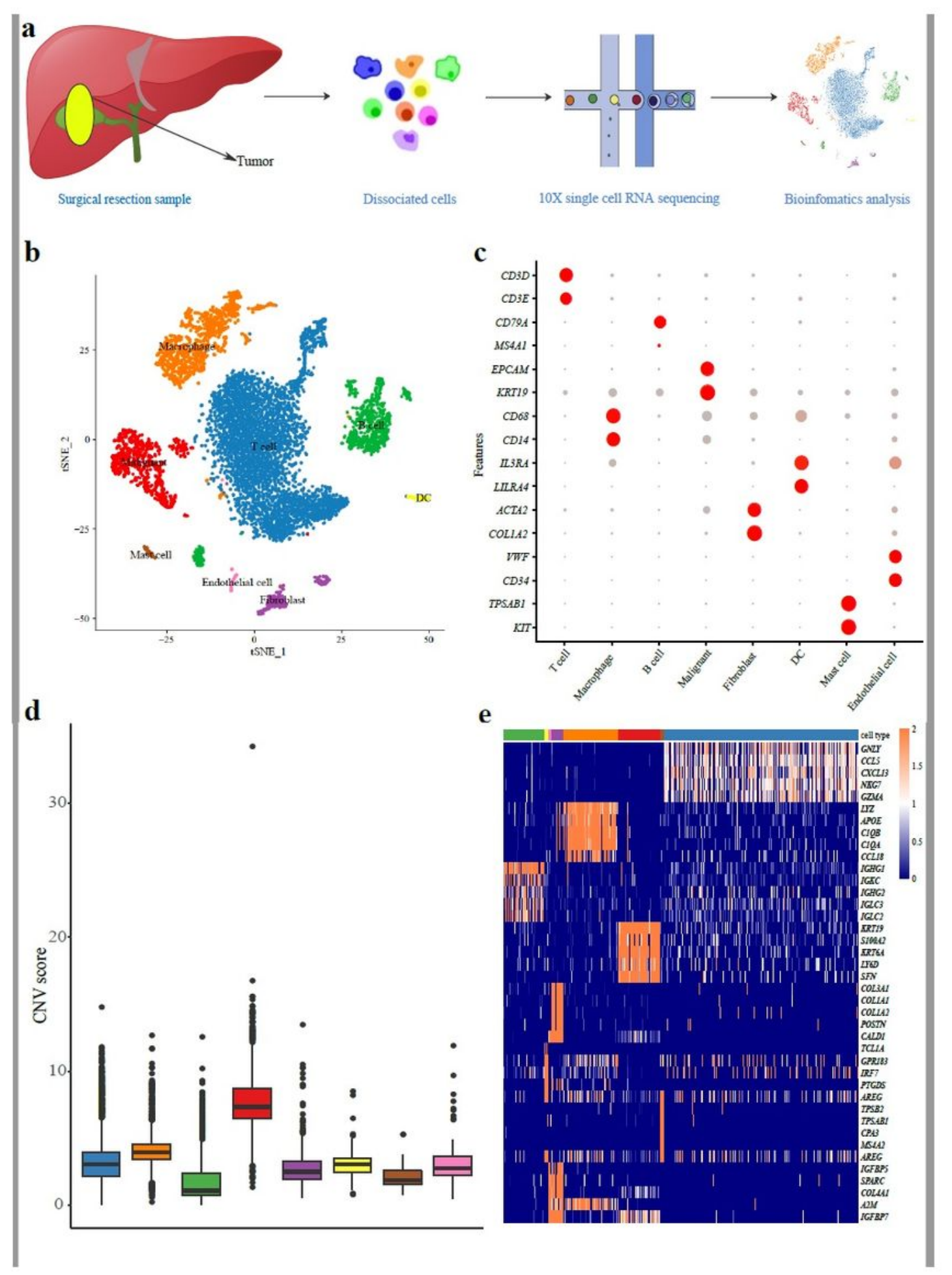

\section{Figure 1}

The overview of cell types distribution in GBC liver metastasis. (a) The workflow of this scRNA-seq study. Metastatic liver tissue was dissociated into single cells and sequenced with 10X platform. (b)The tSNE plot of 8 cell types in this study and each color indicating an associated cell type. (c) Dot plots showing the expression of marker genes in 8 distinct cell types. (d) Box plots of CNV score for each cell type. (e) Heatmap showing the top 5 DEGs in each subtype. 


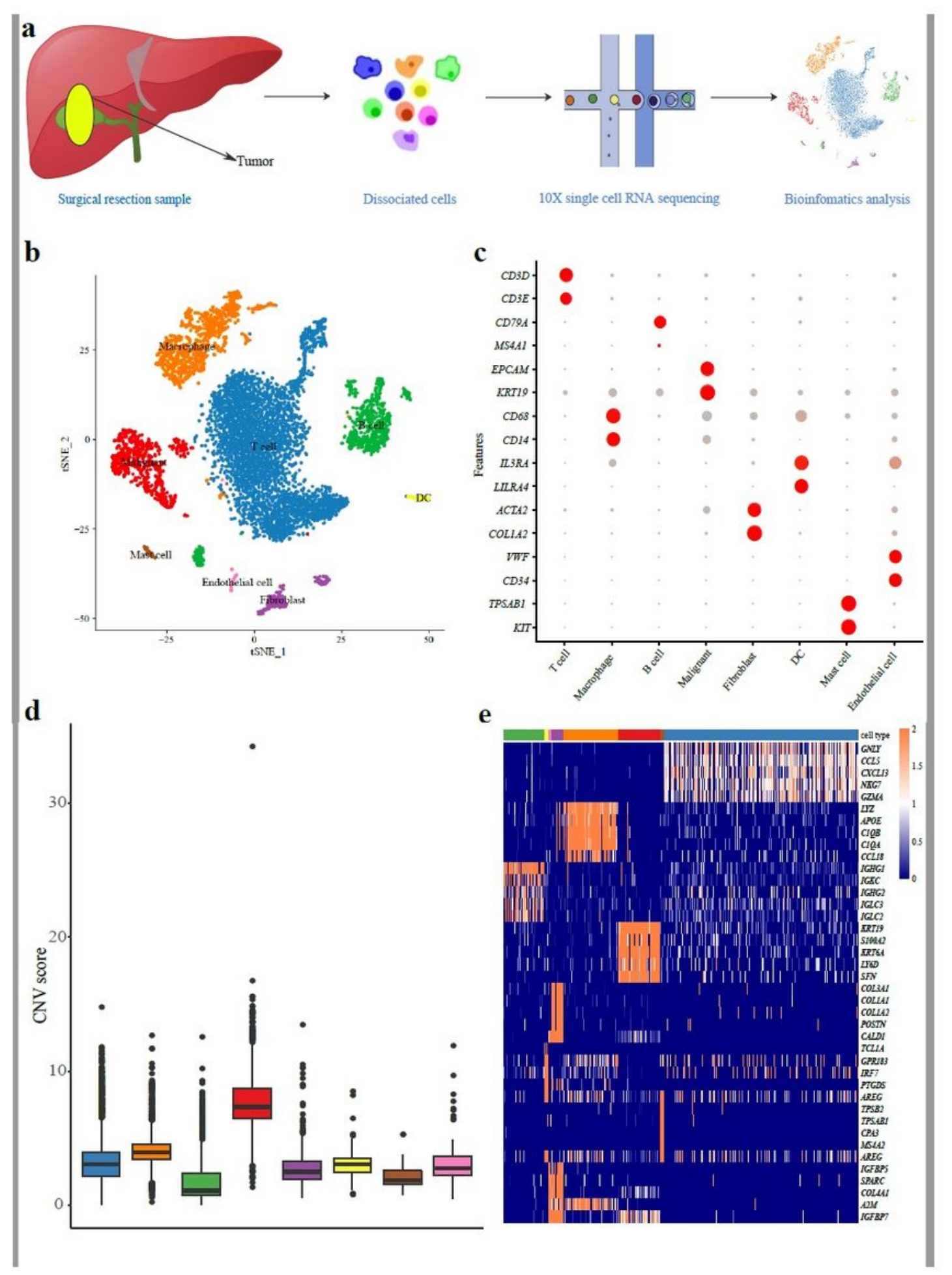

\section{Figure 1}

The overview of cell types distribution in GBC liver metastasis. (a) The workflow of this scRNA-seq study. Metastatic liver tissue was dissociated into single cells and sequenced with 10X platform. (b)The tSNE plot of 8 cell types in this study and each color indicating an associated cell type. (c) Dot plots showing the expression of marker genes in 8 distinct cell types. (d) Box plots of CNV score for each cell type. (e) Heatmap showing the top 5 DEGs in each subtype. 
a

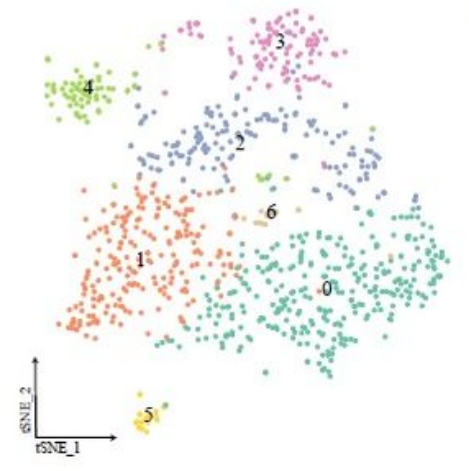

c

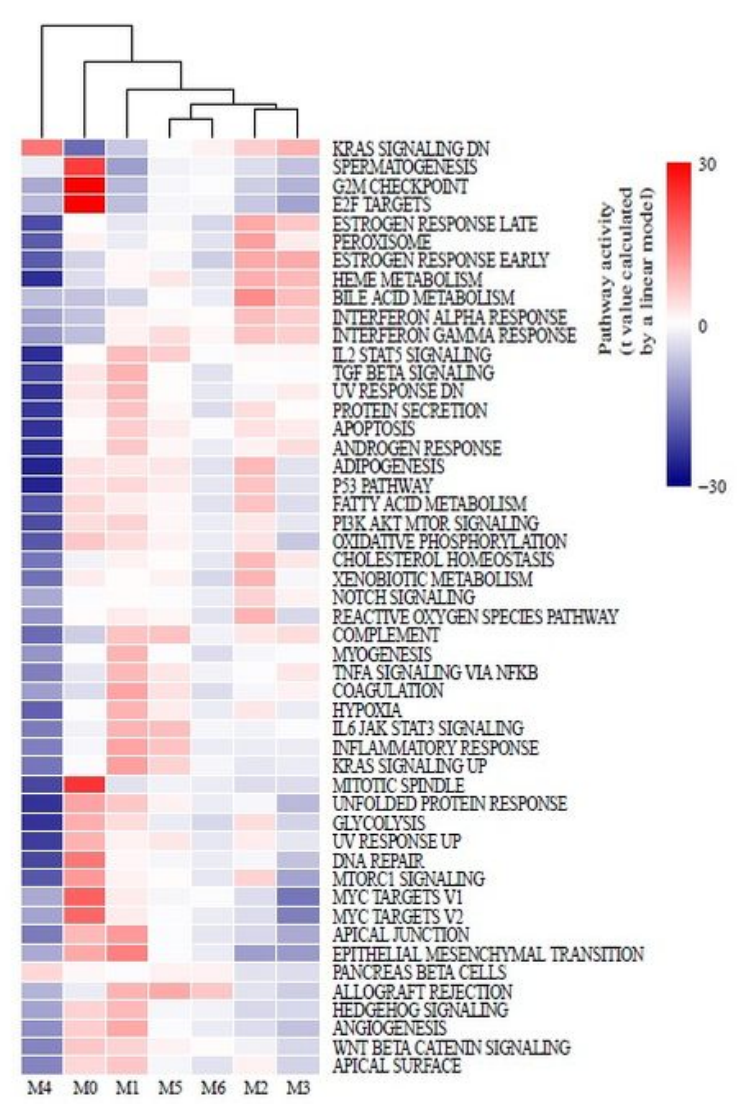

b

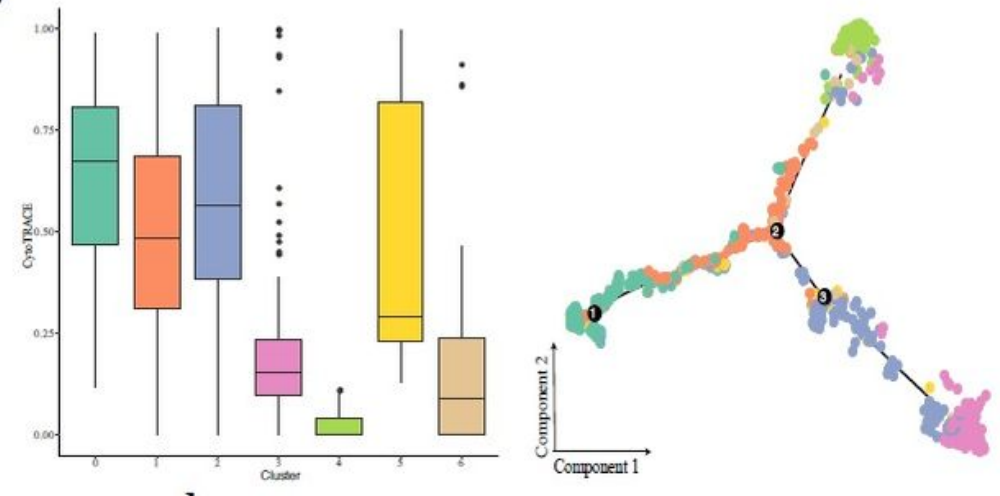

d

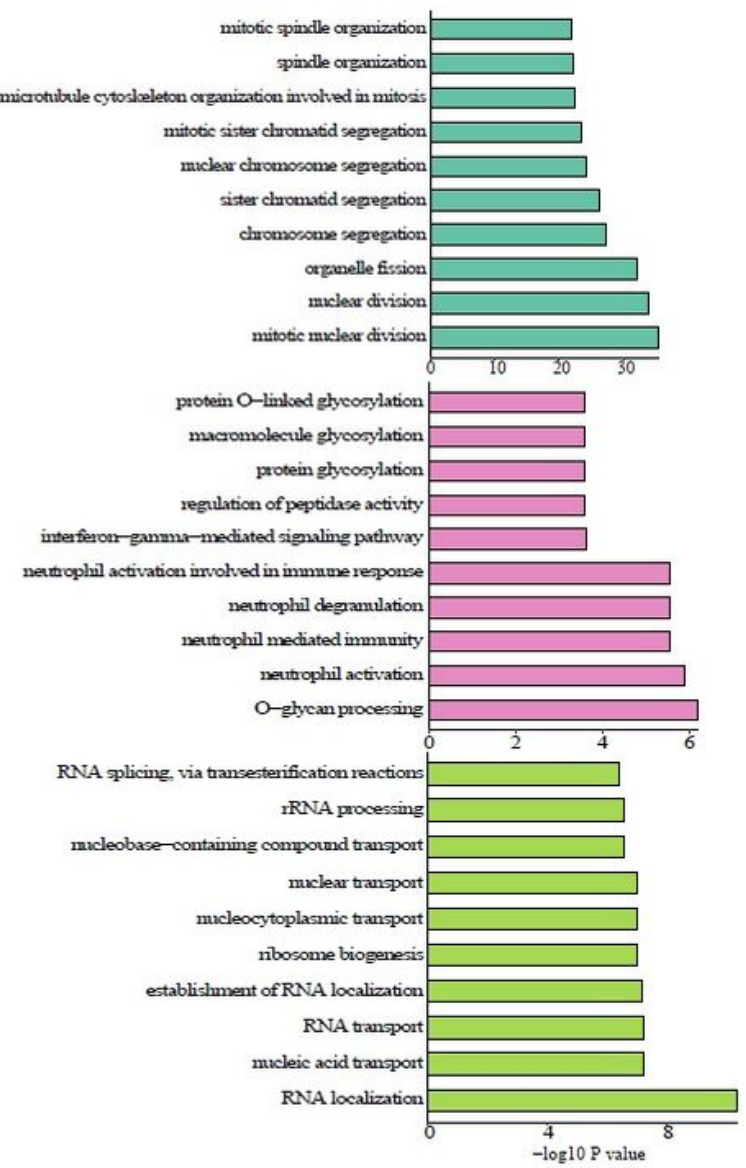

Figure 2

Transcriptomic heterogeneity of malignant cells in metastatic liver tissue. (a) tSNE map showing 7 cancer cell clusters. (b) Boxplot showing transcriptional diversity of cancer cells estimated by CytoTRACE (left) and differentiation trajectory of cancer cells in GBC liver metastasis (right). (c) Differences in 50 hallmark pathway activity scored by GSVA software. $t$ values were calculated by a linear model. (d) GO analysis of DEGs for cluster 0 (top), cluster 3 (middle) and cluster 4 (bottom). FDR<0.05 was considered as significantly enriched. 
a

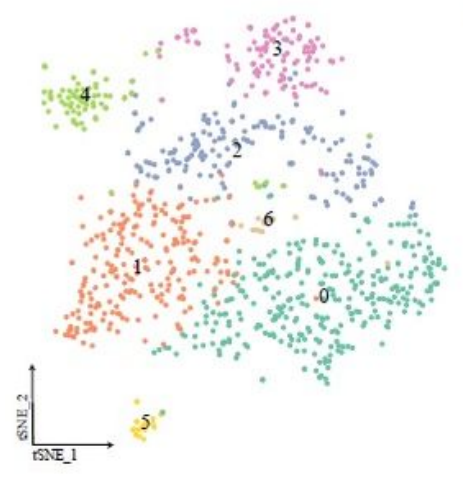

c

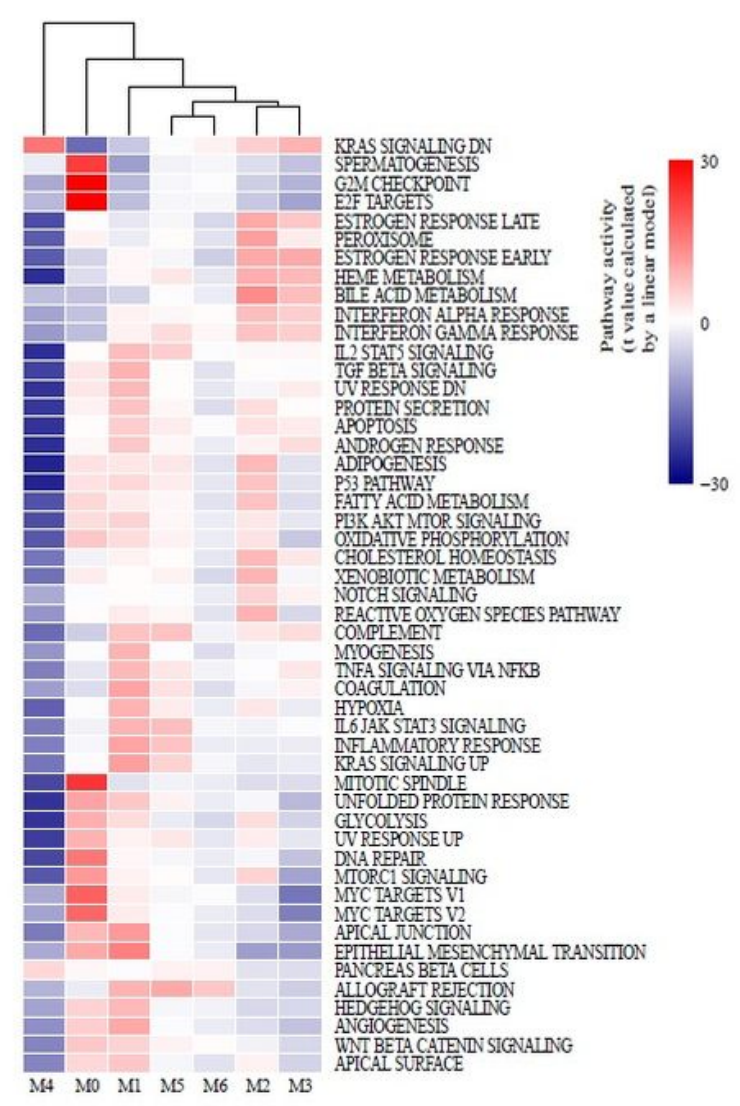

b

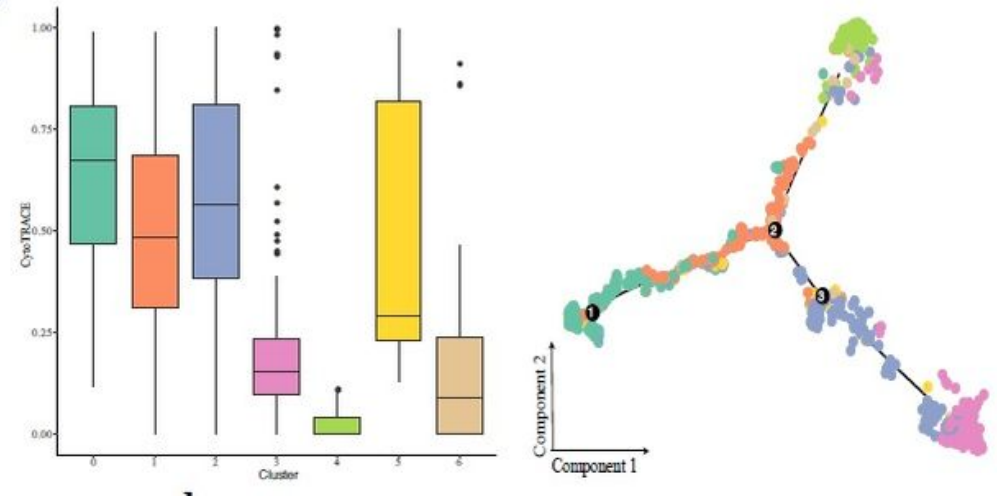

d

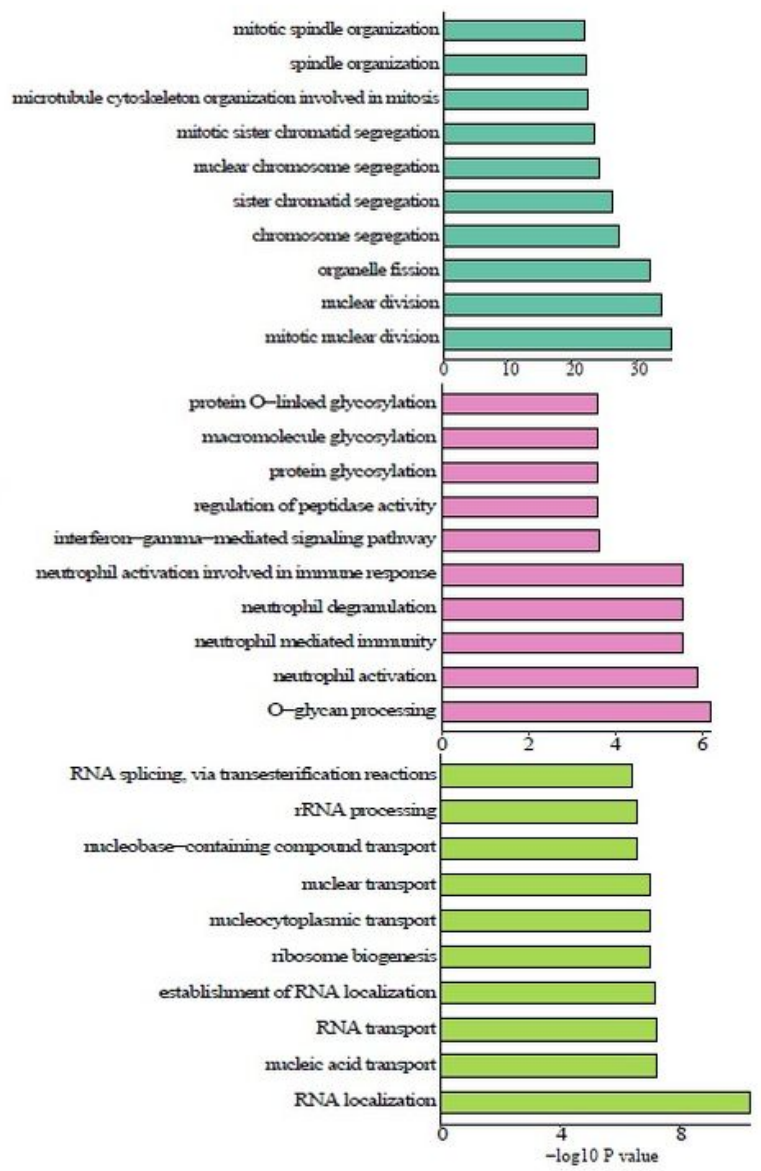

\section{Figure 2}

Transcriptomic heterogeneity of malignant cells in metastatic liver tissue. (a) tSNE map showing 7 cancer cell clusters. (b) Boxplot showing transcriptional diversity of cancer cells estimated by CytoTRACE (left) and differentiation trajectory of cancer cells in GBC liver metastasis (right). (c) Differences in 50 hallmark pathway activity scored by GSVA software. $t$ values were calculated by a linear model. (d) GO analysis of DEGs for cluster 0 (top), cluster 3 (middle) and cluster 4 (bottom). FDR $<0.05$ was considered as significantly enriched. 
a

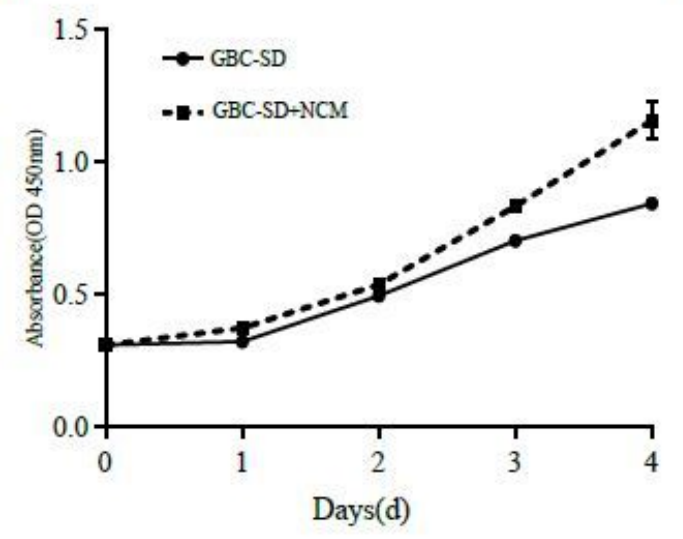

c

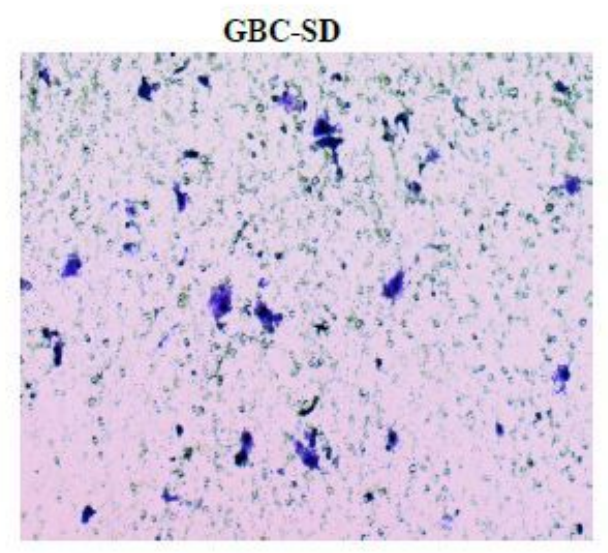

b
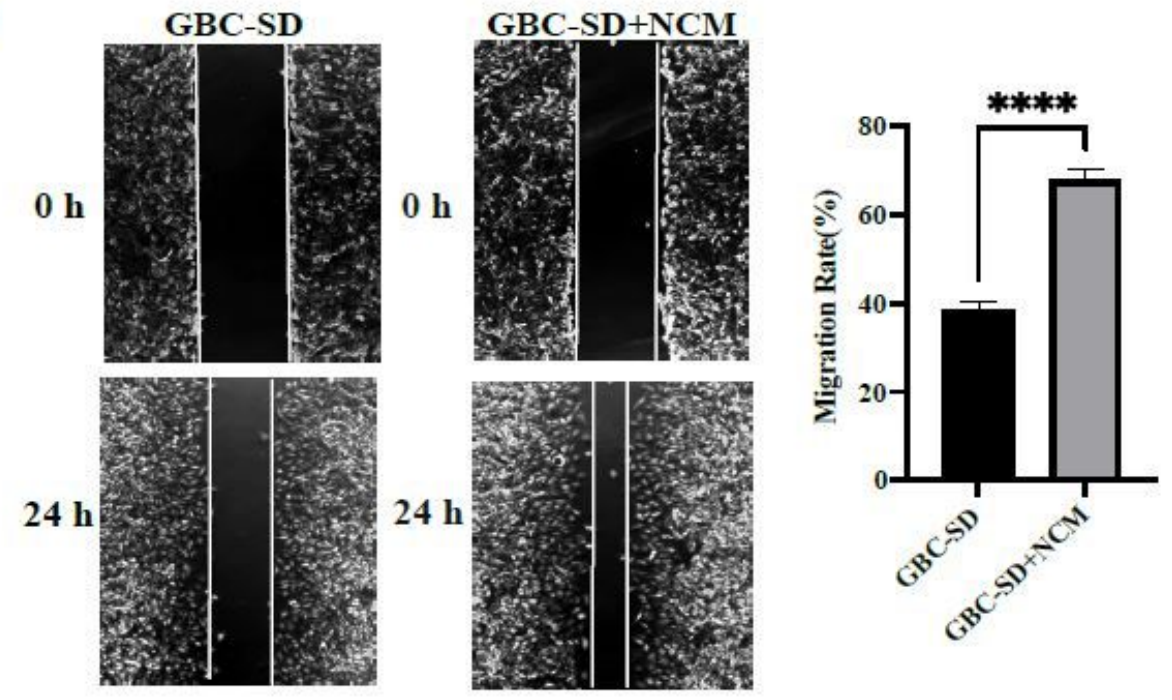
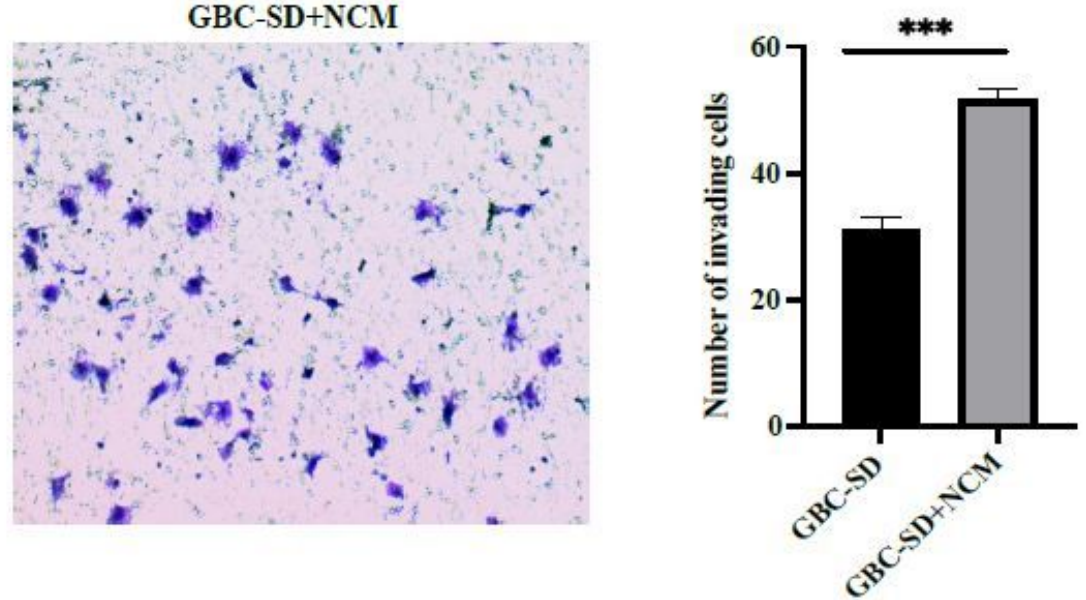

Figure 3

Neutrophils promote proliferation, migration and invasion of GBC cells in vitro. (a) Cell proliferation measured by CCK8 assay. NCM significantly promoted GBC proliferation. (b) Wound-healing migration assays showed that there was a significant increase in the wound closure rate of ICC cells co-cultured with NCM compared with the controls. (c) In vitro invasive assays showed that the number of invasive ICC cells was higher when cells were cultured with NCM than the control. 
a

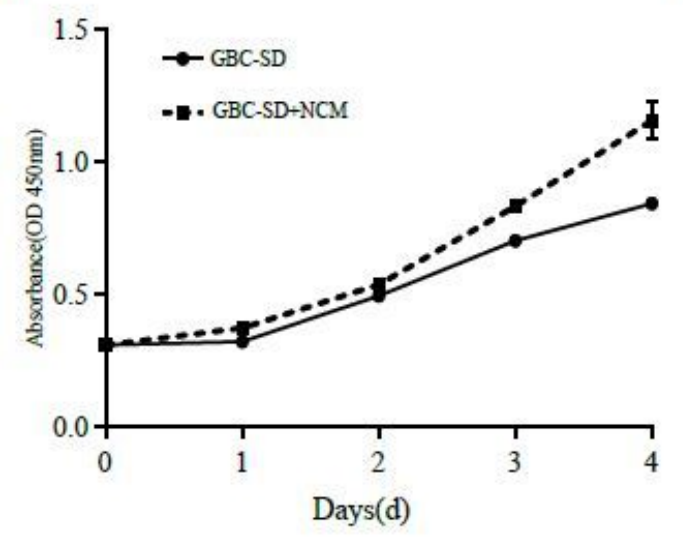

c

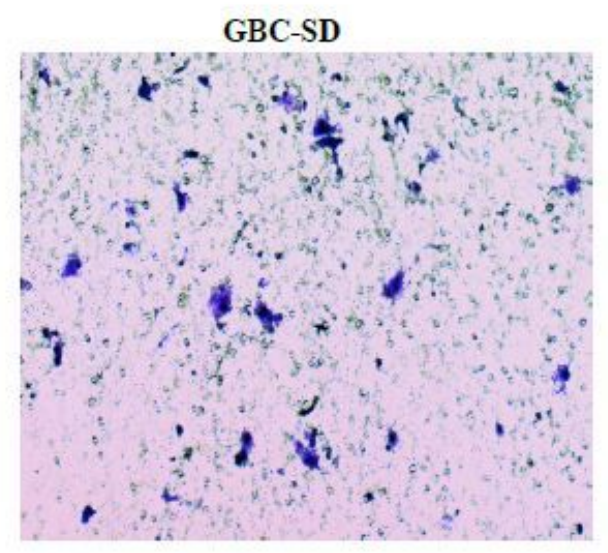

b
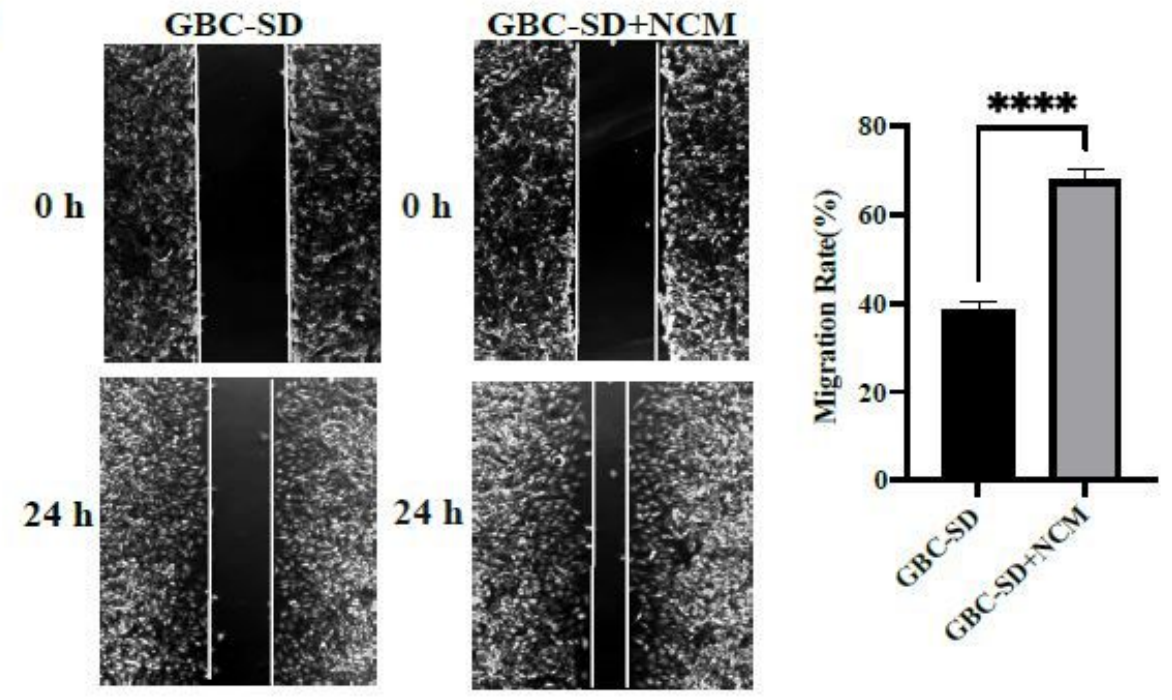
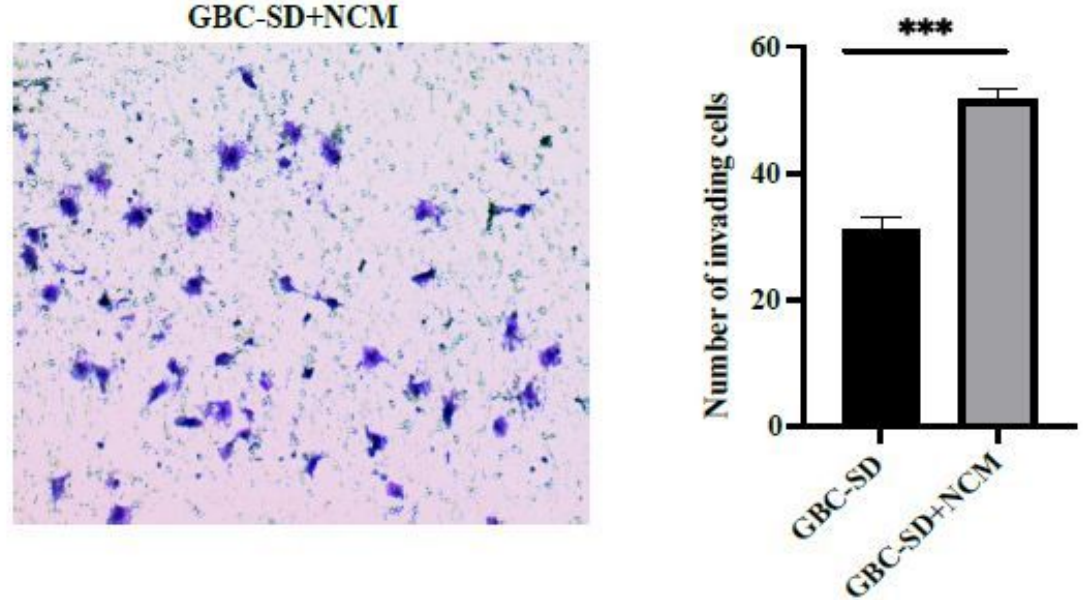

Figure 3

Neutrophils promote proliferation, migration and invasion of GBC cells in vitro. (a) Cell proliferation measured by CCK8 assay. NCM significantly promoted GBC proliferation. (b) Wound-healing migration assays showed that there was a significant increase in the wound closure rate of ICC cells co-cultured with NCM compared with the controls. (c) In vitro invasive assays showed that the number of invasive ICC cells was higher when cells were cultured with NCM than the control. 
a

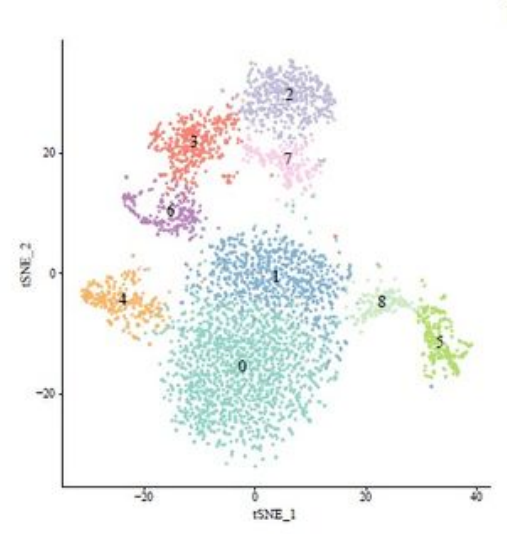

c
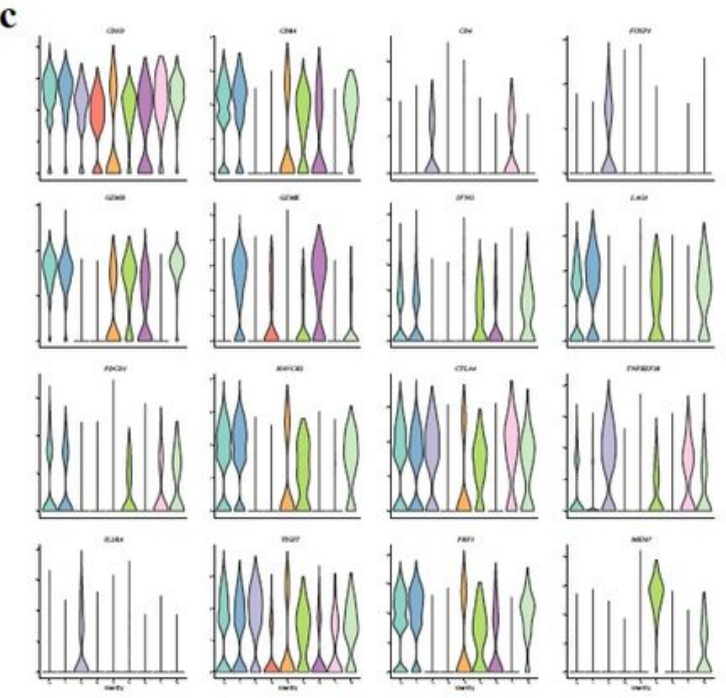

e

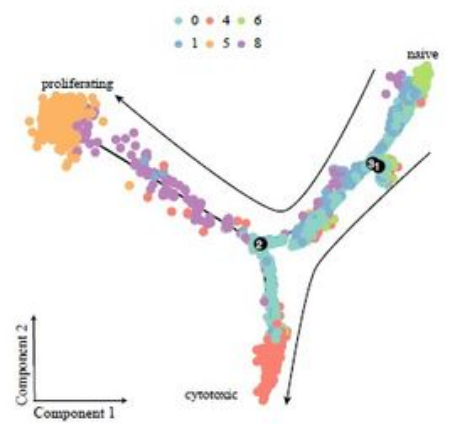

$\mathbf{f}$
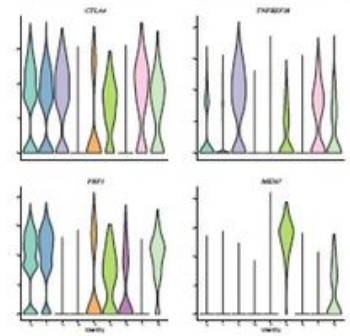

f b
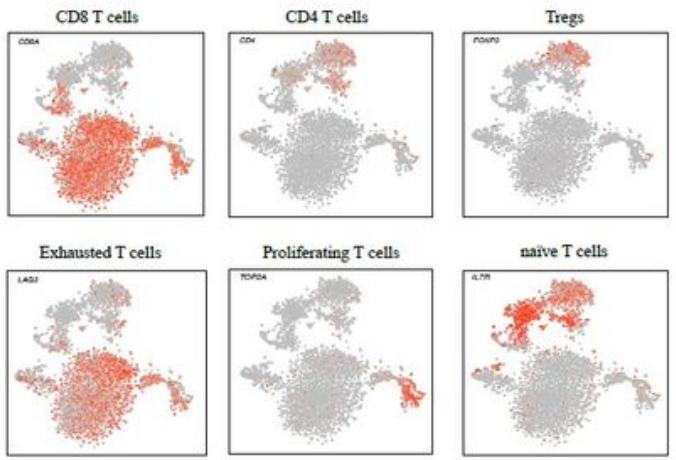

d

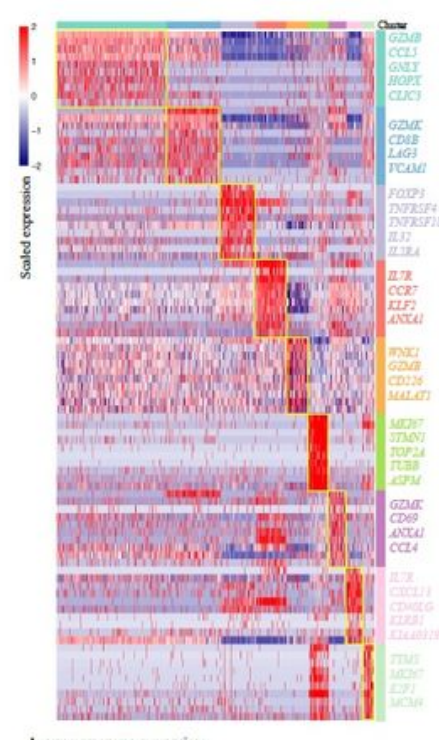

Average ganzyme expression

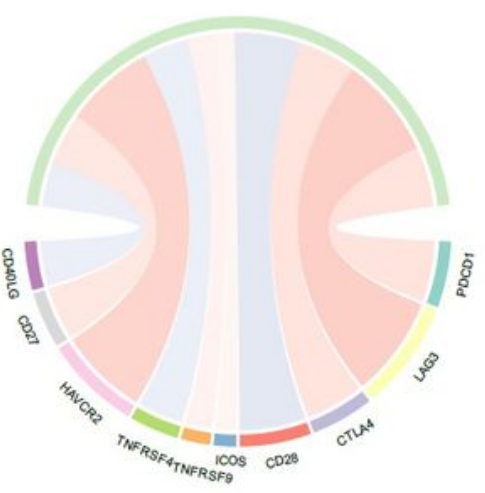

\section{Figure 4}

Distinct subpopulations of infiltrating T cells in the metastatic TME. (a) tSNE plot for 9 T cell subclusters. (b) t-SNE plots showing the expression of marker genes for each cell type. (c)Violin plots of cytotoxic, proliferating, and suppressive genes in distinct T cell subclusters. (d) Heatmap showing the expression of top 10 DEGs in each cluster. (e) Differentiation trajectory of CD8+ cytotoxic T cells. (f) Spearman 
correlation between the activity of CD8+ T cells, as measured by the mean granzyme expression (GZMA, GZMB and GZMH) and known immune checkpoint molecules.

a

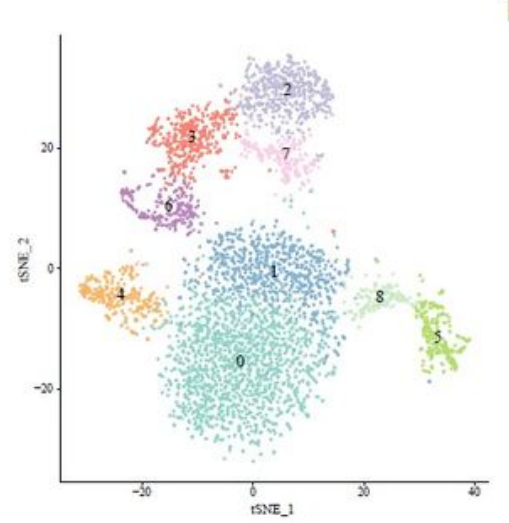

c
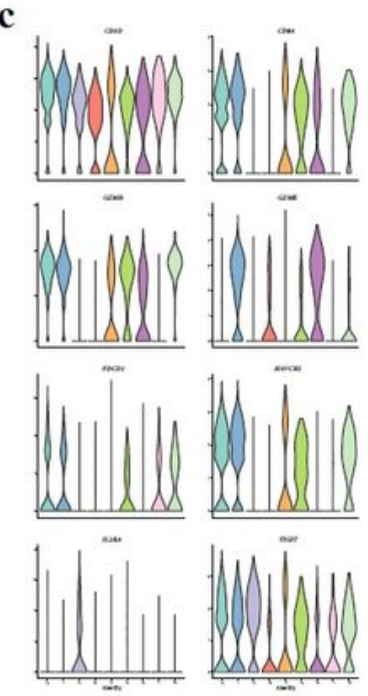

e

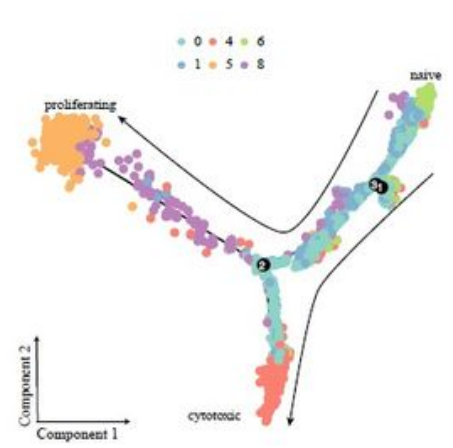

b
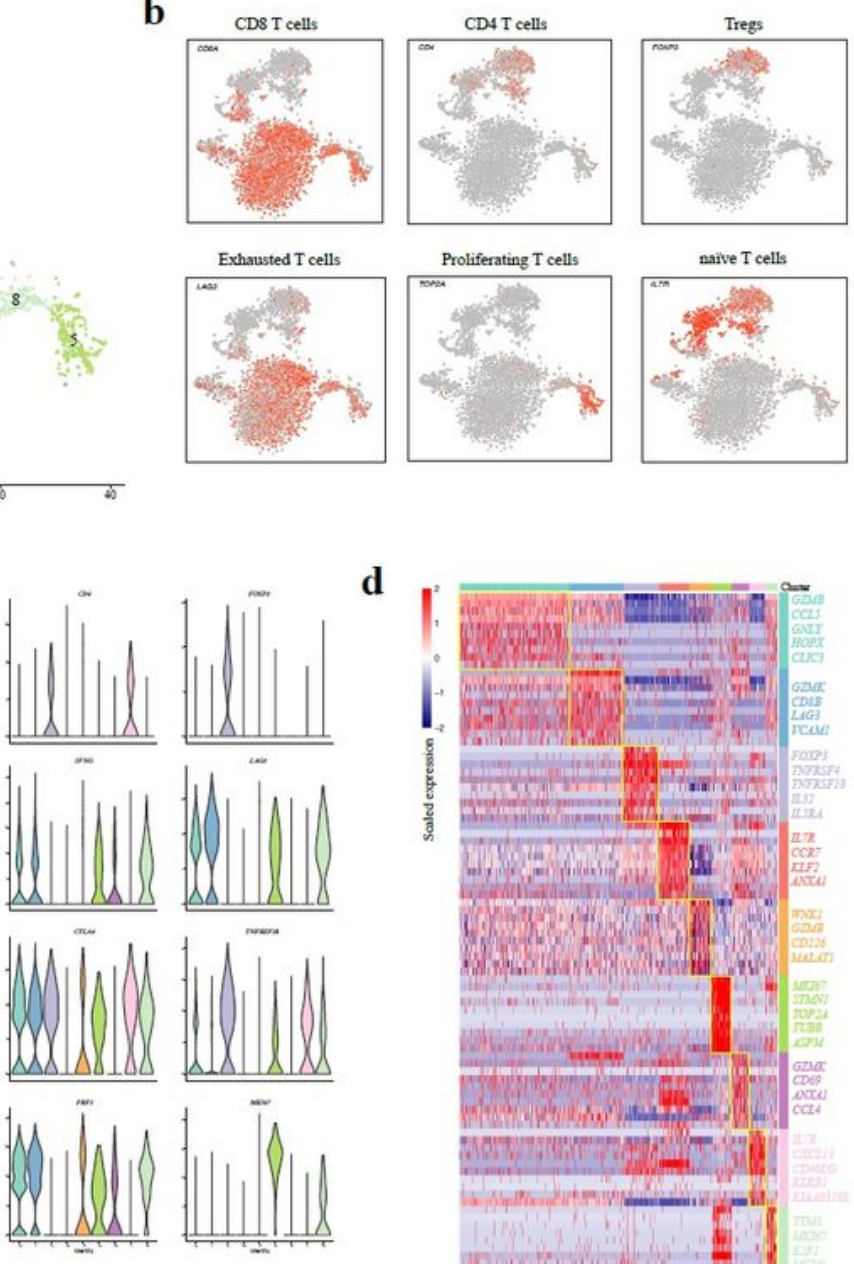

f d
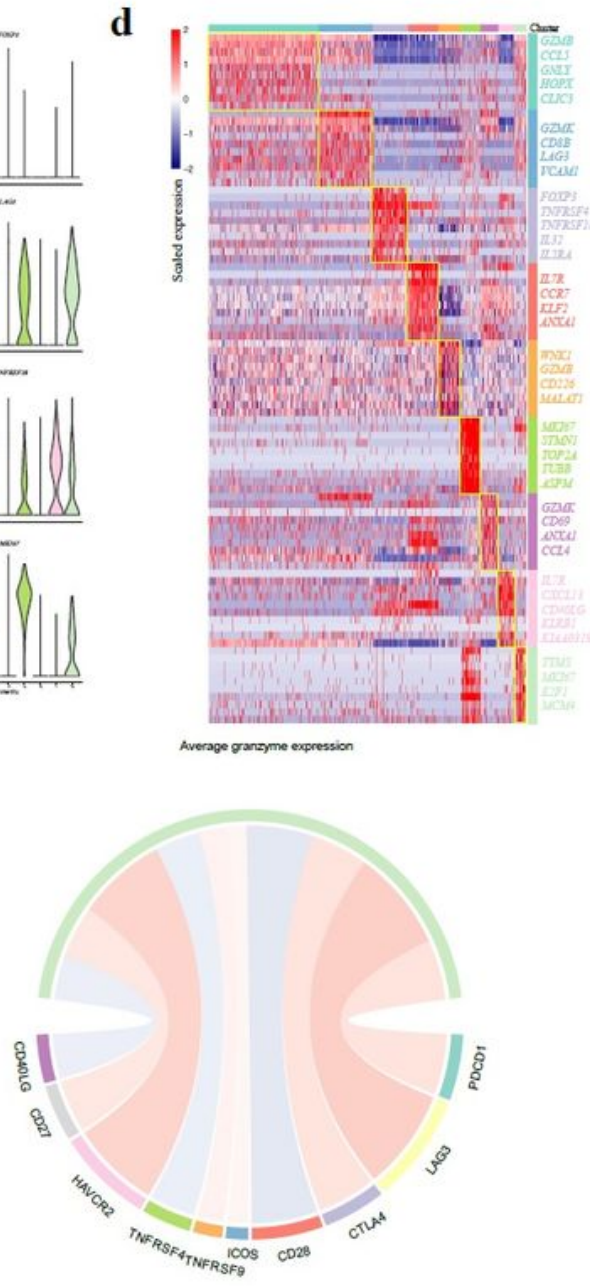

\section{Figure 4}

Distinct subpopulations of infiltrating T cells in the metastatic TME. (a) tSNE plot for 9 T cell subclusters. (b) t-SNE plots showing the expression of marker genes for each cell type. (c)Violin plots of cytotoxic, proliferating, and suppressive genes in distinct T cell subclusters. (d) Heatmap showing the expression of 
top 10 DEGs in each cluster. (e) Differentiation trajectory of CD8+ cytotoxic T cells. (f) Spearman correlation between the activity of CD8+ T cells, as measured by the mean granzyme expression (GZMA, GZMB and GZMH) and known immune checkpoint molecules.

a

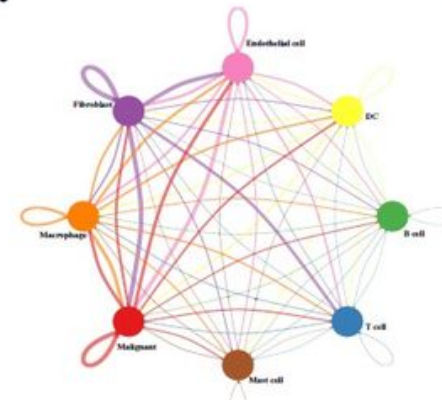

b

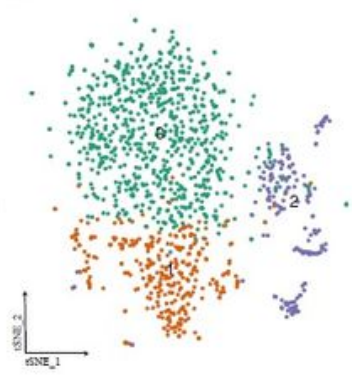

d

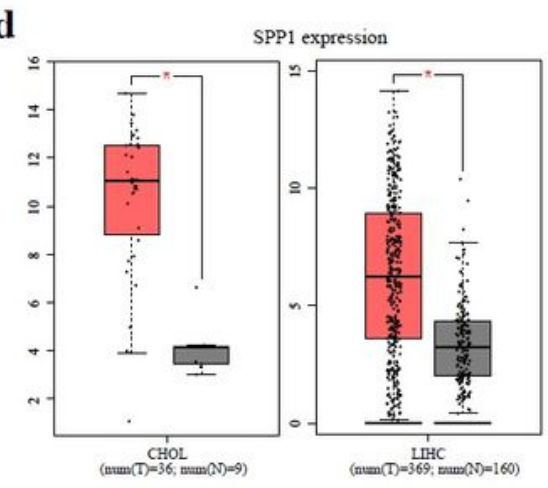

e

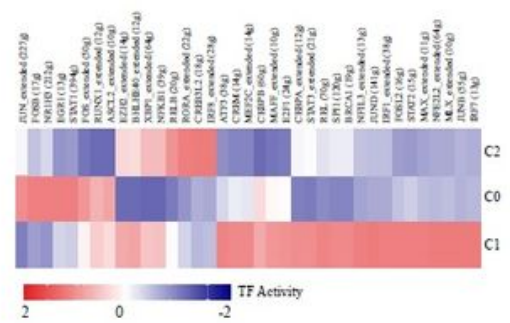

g

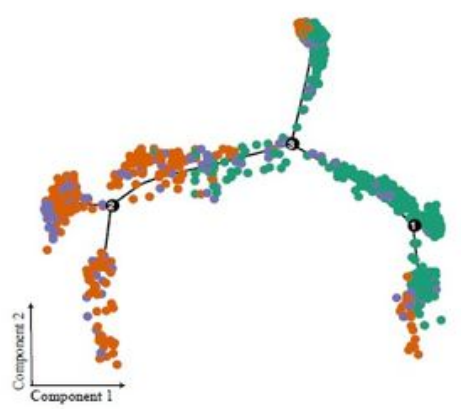

c

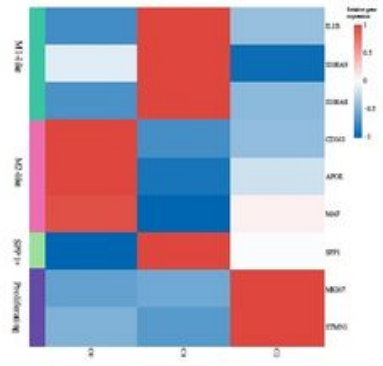

f
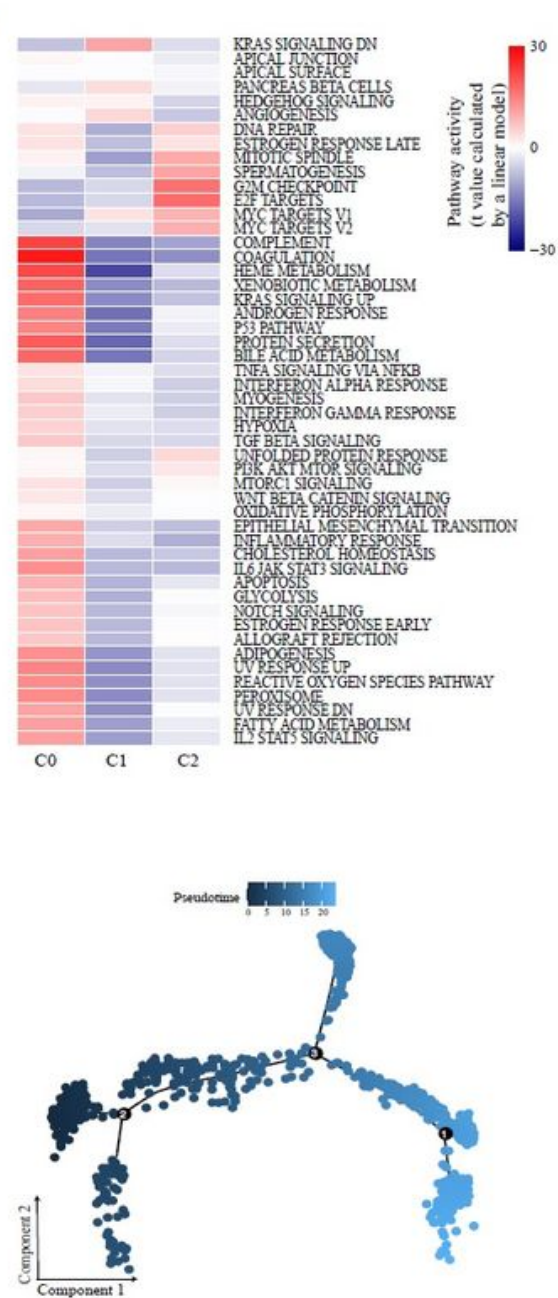

Figure 5

Macrophages emerge M2 polarization in the TME. (a) Interaction network of 8 cell types in the TME constructed by CellPhoneDB. Thicker line means more interaction. (b) tSNE plot of three subclusters of 
macrophages. (c) Average expression of canonical marker genes for macrophage subpopulations. (d) SPP1 expression in TCGA LIHC cohort and CHOL cohort. (e) Heatmap showing the area under the curve (AUC) scores of expression regulation by transcription factors estimated by SCENIC. (f) Heatmap of differences in 50 hallmark pathways scored per cell by GSVA. (G) Differentiation trajectory of macrophages with each color coded for subclusters (left) and pseudotime (right).

$\mathbf{a}$

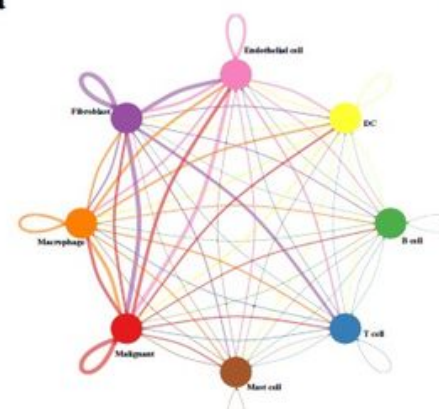

b

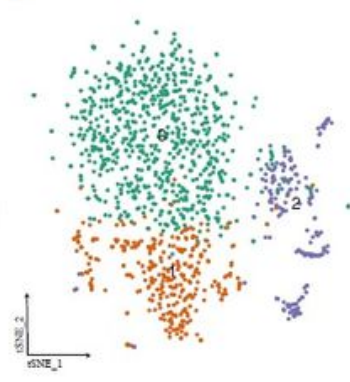

c

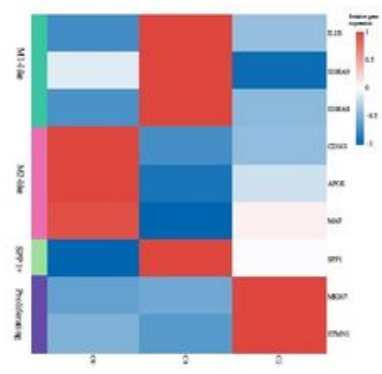

d

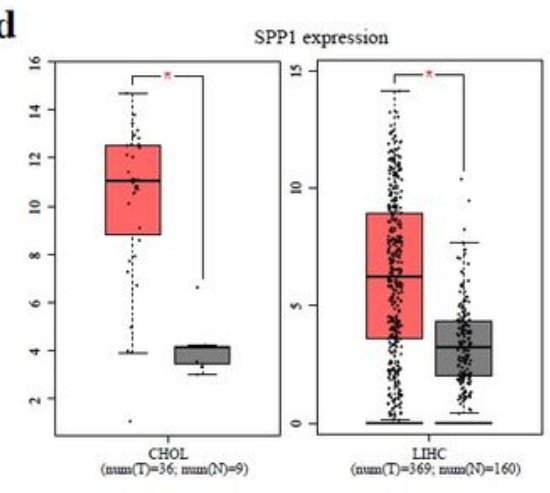

e

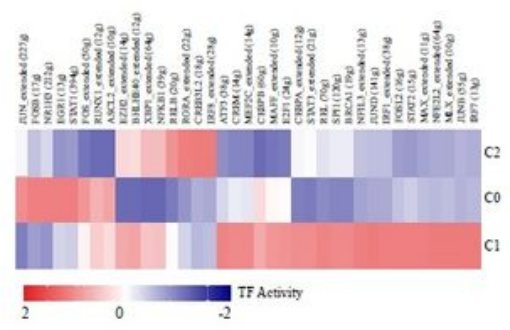

g

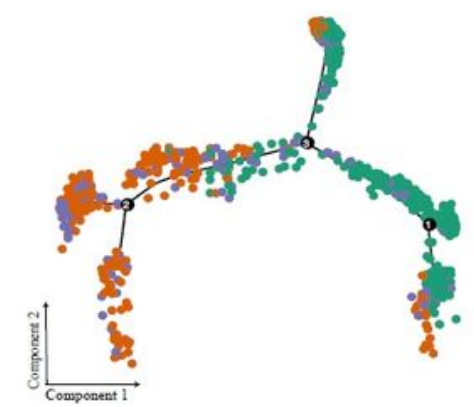

f
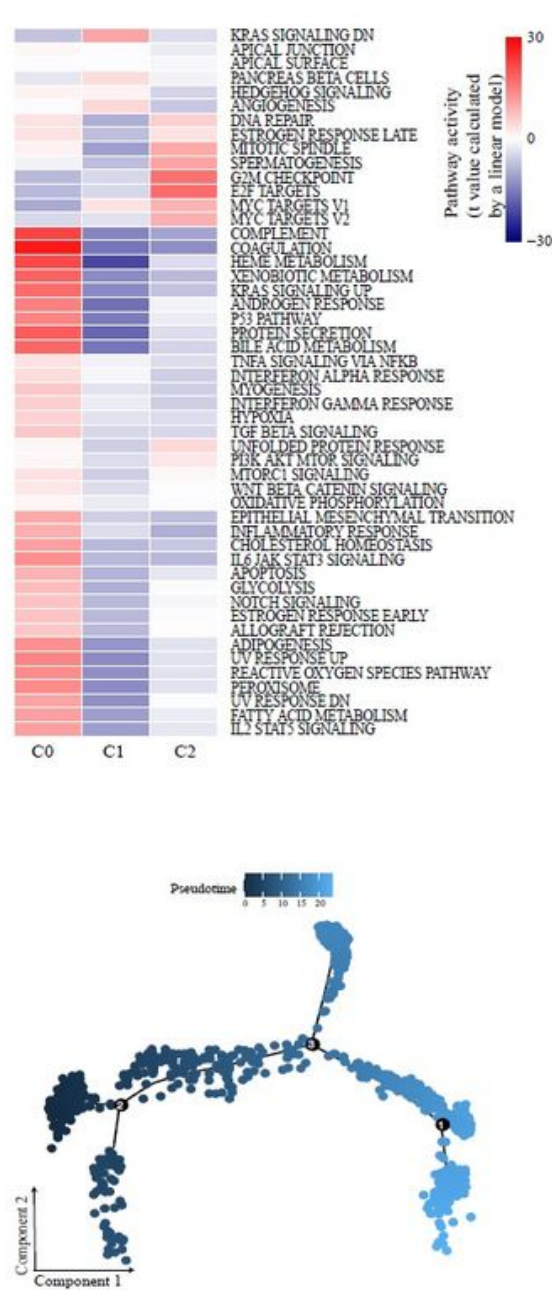

Figure 5 
Macrophages emerge M2 polarization in the TME. (a) Interaction network of 8 cell types in the TME constructed by CellPhoneDB. Thicker line means more interaction. (b) tSNE plot of three subclusters of macrophages. (c) Average expression of canonical marker genes for macrophage subpopulations. (d) SPP1 expression in TCGA LIHC cohort and CHOL cohort. (e) Heatmap showing the area under the curve (AUC) scores of expression regulation by transcription factors estimated by SCENIC. (f) Heatmap of differences in 50 hallmark pathways scored per cell by GSVA. (G) Differentiation trajectory of macrophages with each color coded for subclusters (left) and pseudotime (right).
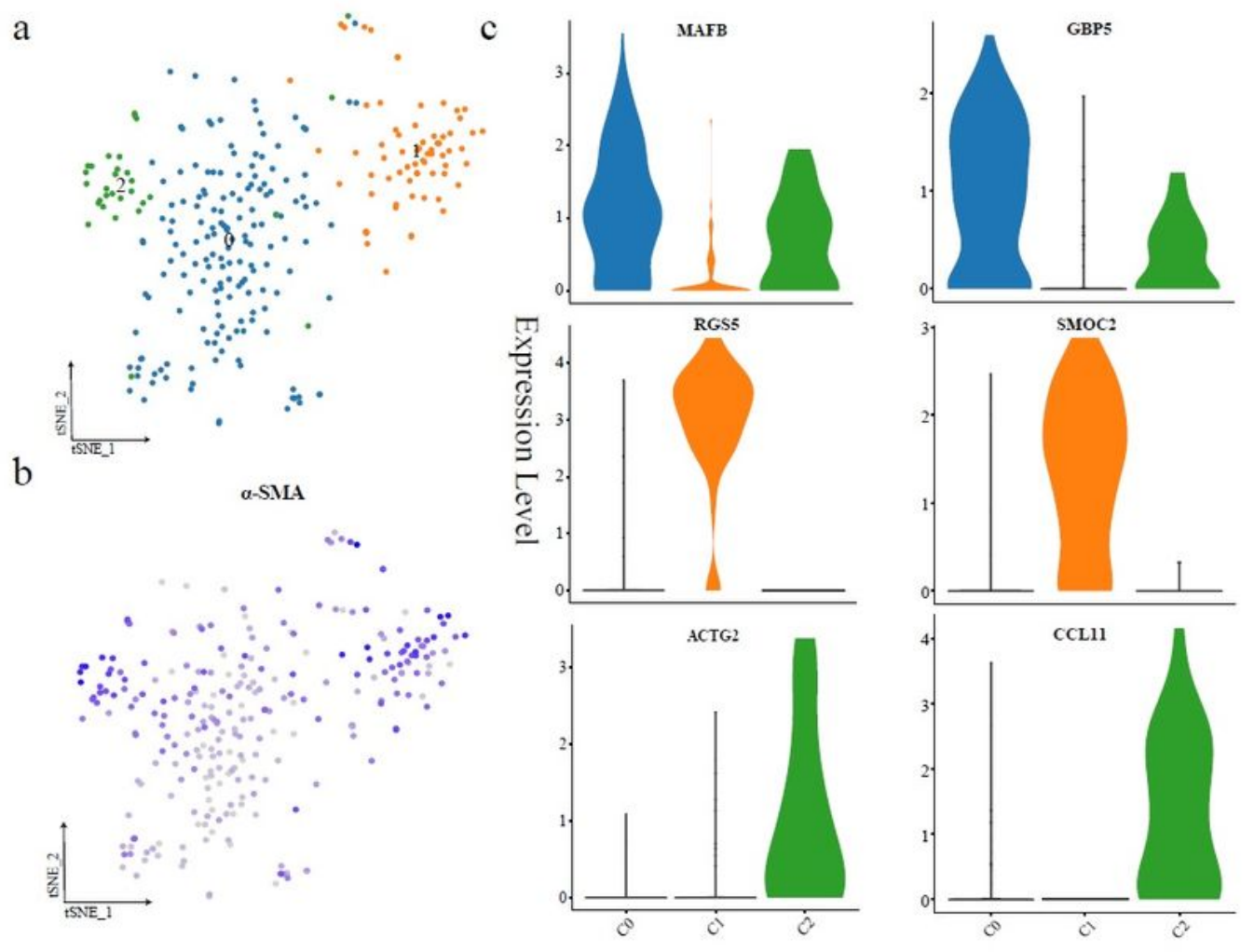

d

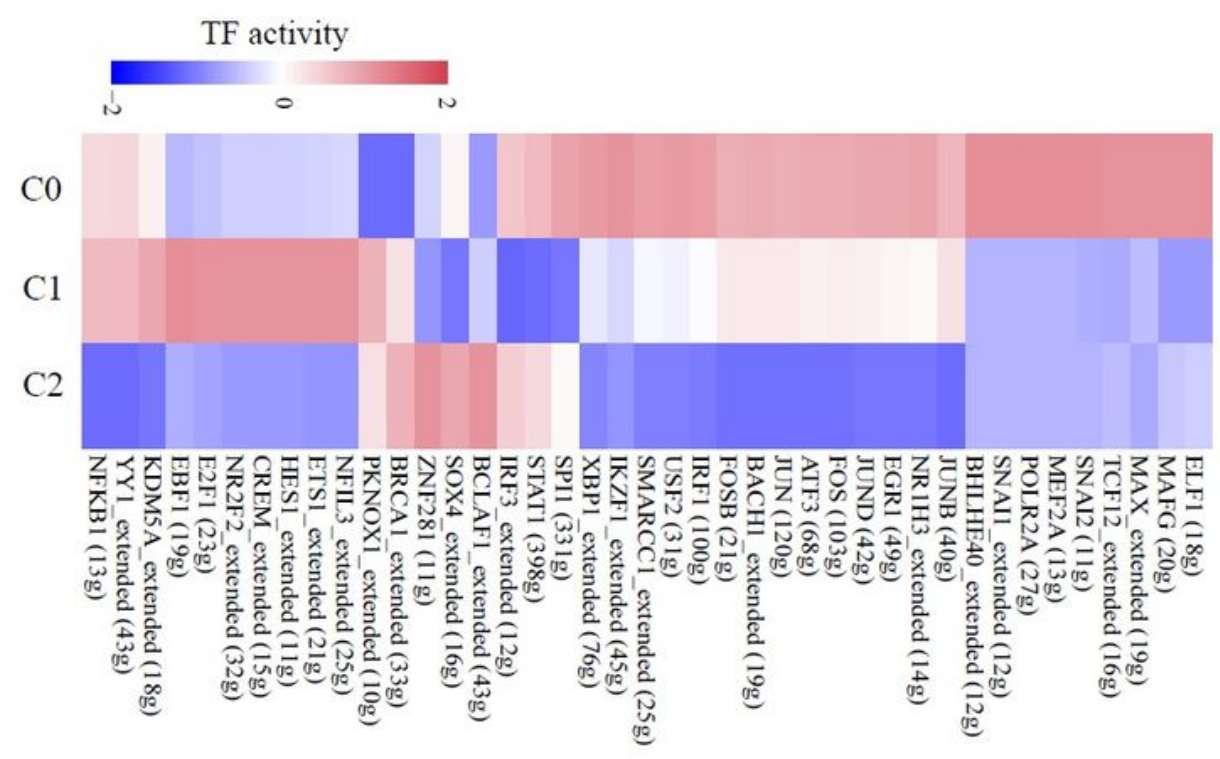


Figure 6

Three distinct fibroblast subpopulations detected in GBC liver metastasis. ( $a$ and b) tSNE plot of three fibroblast subpopulations(a) and the expression levels of a-SMA (ACTA2) (b). (c) Violin plots showing marker genes of three subclusters. (d) Differences of AUC scores in expression regulated by transcription factors (TFs).

a

$\mathrm{b}$

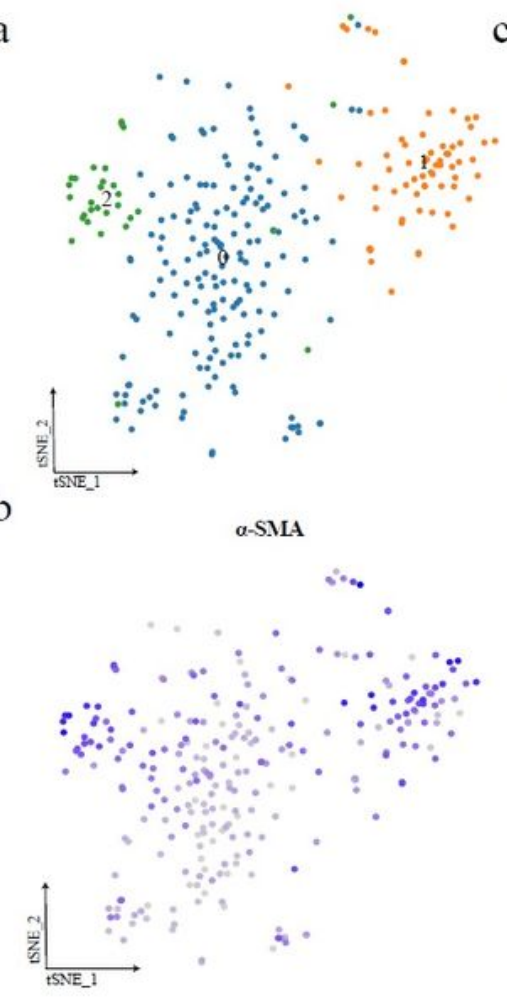

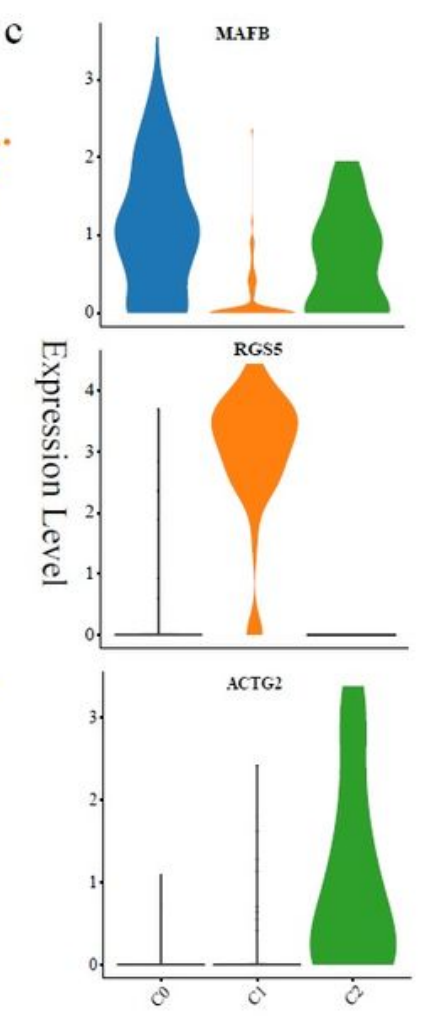
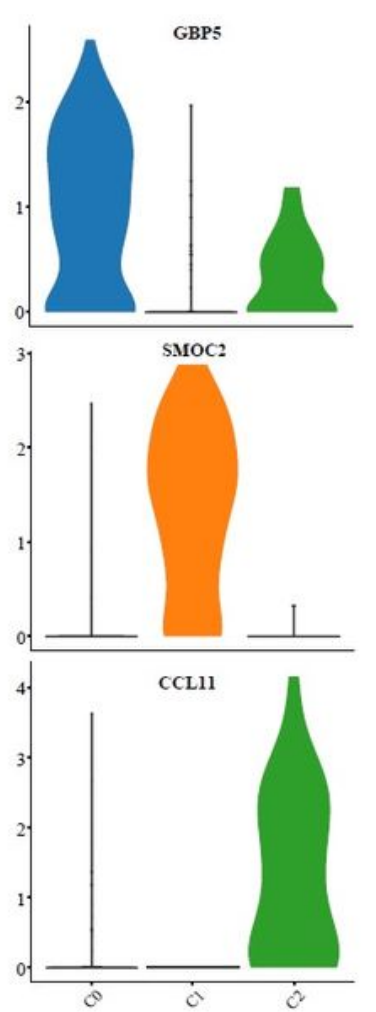

d

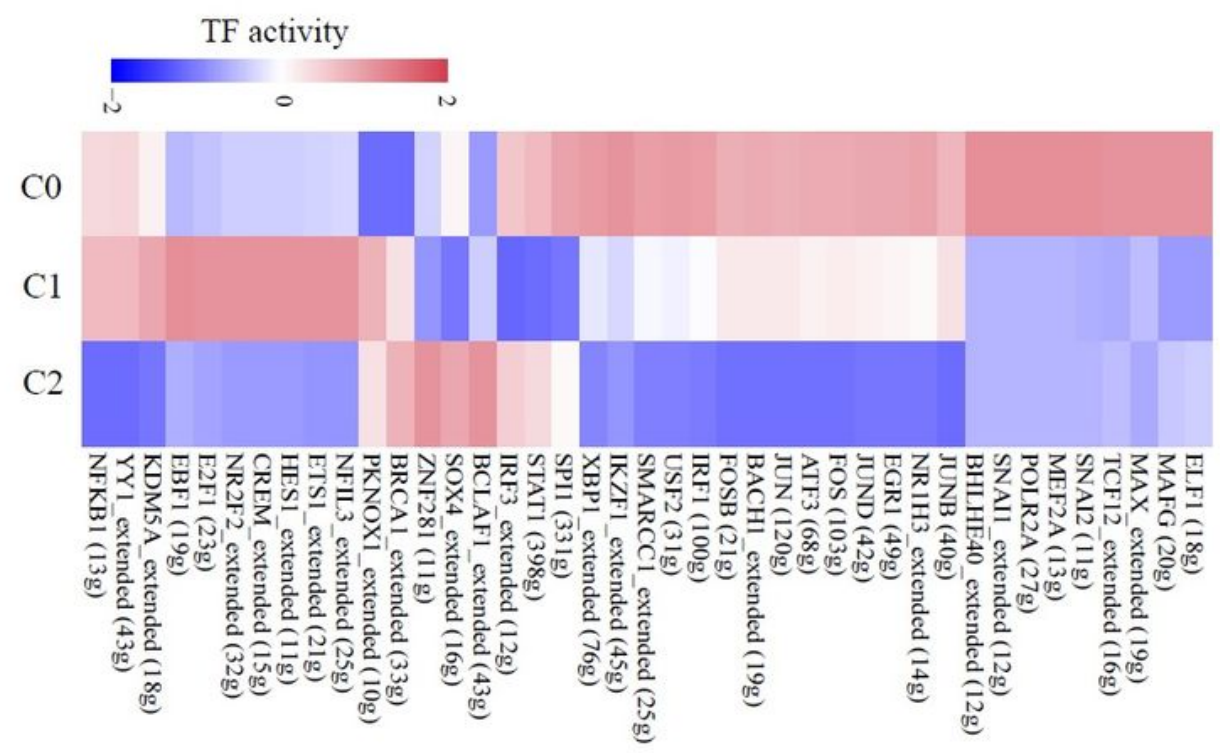

Figure 6 
Three distinct fibroblast subpopulations detected in GBC liver metastasis. ( $a$ and b) tSNE plot of three fibroblast subpopulations(a) and the expression levels of a-SMA (ACTA2) (b). (c) Violin plots showing marker genes of three subclusters. (d) Differences of AUC scores in expression regulated by transcription factors (TFs).

\section{Supplementary Files}

This is a list of supplementary files associated with this preprint. Click to download.

- S1.pdf

- S1.pdf

- S2.pdf

- S2.pdf

- S3.pdf

- S3.pdf

- S4.pdf

- S4.pdf

- S5.pdf

- S5.pdf 